\title{
VALUACIÓN DEL VALOR EN RIESGO DE BONOS CUPÓN CERO EN EL MERCADO FINANCIERO MEXICANO A TRAVÉS DEL MODELO DE VASICEK, CIR Y SIMULACIÓN MONTE CARLO CON SALTOS DE POISSON
}

\section{Fernando Cruz Aranda*}

Centro de Investigación en Finanzas

Tecnológico de Monterrey, Campus Ciudad de México

(Recibido 25 de agosto de 2005, aceptado 17 de febrero 2006)

\section{Resumen}

En este trabajo de investigación se calcula el "Valor en Riesgo", llamado VaR, de un bono cupon cero. Se utiliza la tasa de rendimiento anualizada diaria de cetes a 28 días, en el periodo del 3 de mayo de 2004 al 8 de marzo de 2006 para la economía Mexicana. La tasa corta es guiada por una ecuación diferencial estocástica, en particular, del modelo de Vasicek y CoxIngersoll-Ross (CIR). La estimación de los parámetros es por MCO y MGM respectivamente. Asimismo, se determina la estructura de plazos del bono cupon cero. Finalmente, se calcula el precio del bono por simulaciones Monte Carlo en la que la dinámica de la tasa corta sigue un proceso estocástico del tipo de Vasicek y CIR, donde la incorporan de saltos de Poisson conducen a una serie de conclusiones y recomendaciones.

\section{Abstract}

In this paper, the "Value in Risk" of a bond coupon zero is calculed. Daily rate of return of CETES-28 days in the period from 3 May 2004 to 8 March 2006 is used. Short rate is guided by a stochastic differential equation in the Vasicek model and Cox-Ingersoll-Ross model (CIR). The estimation of the parameters is by OLS and GMM respectively. Also, it is determined the structure of terms of the bond coupon zero. Finally, the bond coupon zero price is calculated by Monte Carlo simulation, the dynamic of short rate follows a stochastic process such as Vasicek and CIR and Poisson jumps are gotten up, it lead us to a series of conclusions and recommendations.

Clasificación JEL : C15, G11, G12

Palabras clave: Valor en riesgo, bono cupón cero, simulación Monte Carlo

* Tecnológico de Monterrey, Campus Ciudad de México. Calle del Puente 222, Col. Ejidos de Huipulco, C. P. 14380, Tlalpan, México, D.F. Teléfono: +52(55)54832254. Correo electrónico: fercruz@itesm.mx

$\mathrm{El}$ autor desea agradecer por los comentarios a realizar. El autor es el único responsable por los errores cometidos en el presente trabajo. 


\section{Introducción}

El dinero o capital, es el medio que permite impulsar el desarrollo de un país a través de la edificación de escuelas, centros de investigación, centros de desarrollo tecnológico, hospitales, fabricas, plantas generadoras de luz, exploración de nuevas fuentes de energía, edificación de nuevos pozos petroleros, sistemas de agua potable y alcantarillado, en general redes de comunicación y telecomunicaciones, fundidoras y el desarrollo de capital humano entre otros rubros. Un buena parte de este dinero es obtenido a través de deuda, lo que nos conduce ha un mercado llamado de bonos, en particular de bonos cupon cero respaldados por el gobierno Federal como lo es el caso de México. En general, existen bonos gubernamentales, corporativos, municipales, etc. Cabe señalar, que el mercado de bonos emitidos por el gobierno ferederal, es decir en México, asciende a varios millones de pesos al mes.

Asimismo, se sabe que desde el siglo XIX el Reino Unido ha utilizado y controlado la tasa corta como un instrumento de tipo económico es decir como un mecanismo para controlar la inflación y que en los mercados se percibe rápidamente. Posiblemente uno de los motivos por los que se ha desarrollado insensantemente y existan en la literatura varias clases de modelos que describen el comportamiento de una tasa corta con reversión a la media entre los que destacan modelos de un solo factor como los desarrollados por Merton (1970), Vasicek (1977), Dothan (1978), CIR (1985), Ho y Lee (1986), Longstaff (1989) y Hull y White (1990).

En este trabajo de investigación se calcula el "Valor en Riesgo", llamado $\mathrm{VaR}$, de un bono cupon cero en la que se se utiliza la tasa de rendimiento anualizada diaria de cetes a 28 días en el periodo del 3 de mayo de 2004 al 8 de marzo de 2006 para la economía Mexicana. Se supone que la tasa corta es guiada por una ecuación diferencial estocástica, en particular, del modelo de Vasicek y Cox-Ingersoll-Ross (CIR). La estimación de los parámetros es por mínimos cuadrados y por el método generalizado de momentos respectivamente. Asimismo, se determina la estructura de plazos del bono cupon cero. Finalmente, se calcula el precio del bono cupon cero por simulaciones Monte Carlo en la que la dinámica de la tasa corta sigue un proceso estocástico del tipo de Vasicek y CIR donde se incorporan saltos de Poisson y que conducen a una serie de conclusiones y recomendaciones.

El documento está organizado de la siguiente forma: En la sección dos se estudia los modelos de la tasa corta de Vasicek y Cox-Ingersoll-Ross (CIR) en la que se expresa la función de densidad de la tasa corta en términos de la función de distribución gamma y la distribución ji-cuadrada no central. En la sección tres se realiza un revisión de medidas de riesgo y se describe la métodología para calcular el valor en riesgo de un bono cupon cero con la tasa corta de Vasicek en particular para cetes a 28 días. En la sección cuatro se realizan simulaciones Monte Carlo donde la tasa corta es guiada por una ecuación diferencial estocástica, en particular, del modelo de Vasicek y Cox-Ingersoll-Ross (CIR) en la que se incorporan saltos de Poisson cuya intensidad del salto es calculada con tasas de rendimiento (eventos) de un día a otro que estuvieron por arriba del $2 \%$ y por abajo del $5 \%$ para el periodo dado. Finalmente, se da una serie conclusiones y recomendaciones del valor en riesgo en portafolios de inversión y del cálculo del VaR utilizando la dinámica de la tasa corta con los modelos ya citados. 


\section{Modelos de tasa corta con reversión a la media: Vasicek y Cox- Ingersoll-Ross (CIR)}

Existen en la literatura varias clases de modelos que describen el comportamiento de una tasa corta con reversión a la media. En particular, si la tasa corta sigue un proceso de la forma:

$$
\mathrm{d} r_{t}=a\left(b-r_{t}\right) \mathrm{d} t+\sigma r_{t}^{\beta} \mathrm{d} W_{t}
$$

donde $a, b$, y $\sigma$ son constantes positivas, $\beta \geq 0$, y $\left\{W_{t}\right\}_{t \geq 0}$ es un movimiento Browniano definido sobre un espacio fijo de probabilidad $(\bar{\Omega}, \mathcal{F}, \mathbb{P})$ junto con su filtración aumentada $\mathbb{F}=\left\{\mathcal{F}_{t}\right\}_{t \geq 0}$, entonces se establece una de las clases más importantes de modelos de tasa corta con reversión a la media y parámetros constantes.

Observe que en la ecuación (1) existe un término aleatorio con varianza $\sigma^{2} r_{t}^{2 \beta}$ por unidad de tiempo, donde $\beta \geq 0$. Las situaciones en las que $\beta=0 \mathrm{y}$ $\beta=\frac{1}{2}$ son de particular interés porque conllevan a modelos que pueden tratarse analíticamente. En particular, el modelo de Vasicek se obtiene cuando $\beta=0$, mientras que el modelo de Cox, Ingersoll y Ross se obtiene cuando $\beta=\frac{1}{2}$. Especificaciones de (1) con valores de $\beta$ distintos 0 y $\frac{1}{2}$ no son muy populares en la literatura debido a la complejidad y dificultades técnicas que presentan en su tratamiento.

La tabla 1 presenta un resumen de los modelos de tasas que se pueden derivar a partir de la ecuación suguiente

$$
\mathrm{d} r_{t}=a\left(b-r_{t}^{\alpha}\right) \mathrm{d} t+\sigma r_{t}^{\beta} \mathrm{d} W_{t},
$$

donde $a, b$, y $\sigma$ son constantes positivas, entre los que se destacan el modelo de Merton (1970), Vasicek (1977), Dothan (1978), CIR (1985), Ho y Lee (1986), Longstaff (1989) y Hull y White (1990).

Tabla 1. Resumen de casos particulares de la ecuación (2).

\begin{tabular}{|c|c|c|c|}
\hline Modelo & Parámetros & $\nu, a_{t}$ & Proceso \\
\hline $\begin{array}{c}\text { Merton } \\
(1970)\end{array}$ & $\alpha=0 \quad \beta=0$ & $\begin{array}{c}\mu+1 \\
a=1\end{array}$ & $\begin{array}{c}\mathrm{d} r_{t}=\mu \mathrm{d} t+\sigma \mathrm{d} W_{t} \\
\mu, \sigma \text { son ctes }\end{array}$ \\
\hline $\begin{array}{c}\text { Vasicek } \\
(1977)\end{array}$ & $\alpha=1 \quad \beta=0$ & $b, a$ & $\begin{array}{c}\mathrm{d} r_{t}=a\left(b-r_{t}\right) \mathrm{d} t+\sigma \mathrm{d} W_{t} \\
a, b, \sigma \text { son ctes }\end{array}$ \\
\hline $\begin{array}{c}\text { Dothan } \\
\text { (1978) }\end{array}$ & $\beta=1$ & & $\mathrm{~d} r_{t}=\sigma r_{t} \mathrm{~d} W_{t}$ \\
$\sigma$ son cte
\end{tabular}


Tabla 1. (continuación)

\begin{tabular}{|c|cc|c|c|}
\hline $\begin{array}{c}\text { CIR } \\
(1985)\end{array}$ & $\alpha=1$ & $\beta=\frac{1}{2}$ & $b, a$ & $\begin{array}{c}\mathrm{d} r_{t}=a\left(b-r_{t}\right) \mathrm{d} t+\sigma \sqrt{r_{t}} \mathrm{~d} W_{t} \\
a, b, \sigma \text { son ctes }\end{array}$ \\
\hline $\begin{array}{c}\text { Ho y Lee } \\
(1986)\end{array}$ & $\alpha=0$ & $\beta=0$ & $2, h_{t}$ & $\begin{array}{c}\mathrm{d} r_{t}=h_{t} \mathrm{~d} t+\sigma \mathrm{d} W_{t} \\
\sigma \text { es constante }\end{array}$ \\
\hline $\begin{array}{c}\text { Longstaff } \\
(1989)\end{array}$ & $\alpha=\frac{1}{2}$ & $\beta=\frac{1}{2}$ & $b, a$ & $\begin{array}{c}\mathrm{d} r_{t}=a\left(b-\sqrt{r}_{t}\right) \mathrm{d} t+\sigma \sqrt{r_{t}} \mathrm{~d} W_{t} \\
a, b, \sigma \text { son ctes }\end{array}$ \\
\hline $\begin{array}{c}\text { Hull y White } \\
(1990)\end{array}$ & $\alpha=1$ & $\beta=0$ & $b_{t}, a$ & $\begin{array}{c}\mathrm{d} r_{t}=a\left(b_{t}-r_{t}\right) \mathrm{d} t+\sigma \mathrm{d} W_{t} \\
b_{t}, \sigma \text { son función del tiempo }\end{array}$ \\
\hline
\end{tabular}

En esta investigación se abordara los modelos de Vasicek y Cox Ingersoll y Ross, con el objeto de calcular la estructura de plazos de tasas de interés y el valor en riesgo, $\mathrm{VaR}$, de Bonos cupon cero.

\subsection{Modelo de tasa corta de Vasicek}

A continuación se presenta los fundamentos del modelo de Vasicek así como algunas de las características del modelo de la tasa corta con reversión a la media y la estimación de sus parámetros utilizando un conjunto de datos de interes en particular para determinar la estructura de plazos de la tasa. Para observar el comportamiento de la curva de rendimiento, el precio del bono cupon cero y la tasa forward instantánea se gráfica en cada uno de éstos en función de los parámetros a y $b$, y fijando el plazo $T-t$, la volatilidad $\sigma$ y una $r_{t}$. En este caso $a \in\left[\xi_{1}, \xi_{2}\right]$ y $b \in\left[\xi_{1}, \xi_{2}\right]$ de tal forma que se encuentren alrededor de los valores de los parámetros estimados para un conjunto de datos del sector financiero Mexicano, como es el caso.

\subsubsection{Fundamentos del modelo de Vasicek}

En el modelo de Vasicek (1977) se estudia, como caso particular, la dinámica de una tasa corta que presenta reversión de la media hacia un valor constante. Este comportamiento se observa, en muchos casos, cuando se analizan series de tiempo de tasas de corto plazo. A continuación se formaliza la noción de reversión a la media a través de un proceso de Orstein-Uhlenbeck.

Sea $\left\{W_{t}\right\}_{t>0}$ un movimiento Browniano definido sobre un espacio fijo de probabilidad $(\Omega, \mathcal{F}, \mathbb{P})$ y sea $\mathbb{F}=\left\{\mathcal{F}_{t}\right\}_{t \geq 0}$ su filtración aumentada, la cual representa la información del mercado disponible hasta el tiempo $t$. En el modelo 
de Vasicek la tasa corta, $r_{t}$ es conducida por la siguiente ecuación diferencial estocástica:

$$
\mathrm{d} r_{t}=a\left(b-r_{t}\right) \mathrm{d} t+\sigma \mathrm{d} W_{t},
$$

donde $a, b$ y $\sigma$ son constantes positivas y conocidas. En este caso, como puede observarse, $W_{t}$ es la única fuente de incertidumbre. En la especificación exógena de la dinámica estocástica de la tasa corta, expresada en (3), $r_{t}$ es forzada a moverse, en promedio, hacia un nivel de largo plazo $b$ a una velocidad $a$. Si la tasa corta está por arriba de $b$, ésta es forzada a moverse, en promedio, hacia abajo al nivel $b \mathrm{y}$, viceversa, si la tasa corta está por abajo de $b$, ésta es forzada a moverse, en promedio, hacia arriba al nivel $b$.

Ahora, observe que la ecuación (3) es una notación simplificada de la integral estocástica

$$
\begin{aligned}
r_{t}-r_{s} & =\int_{s}^{t} a\left(b-r_{u}\right) \mathrm{d} u+\int_{s}^{t} \sigma \mathrm{d} W_{u} \\
& =a b(t-s)-\int_{s}^{t} a r_{u} \mathrm{~d} u+\sigma \int_{s}^{t} \mathrm{~d} W_{u}
\end{aligned}
$$

ya que el objeto de estudio del cálculo estocástico es la integral estocástica.

\subsubsection{Ecuación diferencial parcial del comportamiento de un bono con tasa corta conducida por el modelo de Vasicek}

El modelo de Vasicek forma parte de los llamados modelos de equilibrio general debido al uso de condiciones de no arbitraje para caracterizar el precio de un bono cupón cero a un plazo dado. En esta sección, bajo los supuestos de equilibrio general y tasa corta neutral al riesgo, se resuelve la ecuación diferencial parcial parabólica del cornportamiento del precio de un bono cupón cero.

En lo que sigue, el precio de bono cupón cero que se coloca en $t$ y que al vencimiento $T$ paga una unidad monetaria se denotará mediante

$$
B=B\left(r_{t}, t ; T\right),
$$

o en forma más simple como $B=B(t, T)$. En el caso del modelo de Vasicek, el precio $B$ de un cupón cero satisface:

$$
\frac{\partial B}{\partial t}+\frac{1}{2} \sigma^{2} \frac{\partial^{2} B}{\partial r^{2}}+a\left(b-r_{t}\right) \frac{\partial B}{\partial r_{t}}-r_{t} B=0 .
$$

La condición final corresponde al pago en el vencimiento del bono, que por simplicidad se supone de una unidad monetaria, es decir $B(T, T)=1$. Las condiciones de frontera dependen de $a, b, \sigma \mathrm{y}$, por supuesto de, $r_{t}$.

\subsubsection{Solución de la ecuación diferencial parcial del comportamiento de un bono con tasa corta conducida por el modelo de Vasicek}

Dado que la ecuación (5) no cuenta con derivadas parciales cruzadas, se supone una solución en variables separables de la siguiente forma:

$$
B(t, T)=e^{A(t, T)-r_{t} D(t, T)} .
$$


Observe que en la fecha de vencimiento, $A(T, T)=0$ y $D(T, T)=0$, ya que $B(T, T)=1$.

La selección de una solución de la forma (6) permite transformar una ecuación diferencial parcial parabólica de segundo orden, en un sistema de dos ecuaciones diferenciales ordinarias de primer orden. El sistema está acoplado de tal manera que primero se determina que

$$
D(t, T)=\frac{1-e^{-a(T-t)}}{a}
$$

y, posteriormente, con base en la solución de (7), se obtiene

$$
A(t, T)=\frac{1}{a^{2}}(D(t, T)-T+t)\left(a^{2} b-\frac{1}{2} \sigma^{2}\right)-\frac{\sigma^{2} D(t, T)^{2}}{4 a},
$$

donde $a, b$ y $\sigma$ son parámetros a definir a partir de un conjunto de datos.

\subsubsection{Curva de rendimiento del modelo de Vasicek}

En esta sección, a partir de los precios del bono para diferentes vencimientos, $B(t, T), T \geq t$, se genera la estructura de plazos de la tasa de interés, $R(t, T)$, mediante la relación

$$
R(t, T)=-\frac{1}{T-t} \ln B(t, T) .
$$

Así, la sustitución de (7), (8) en (9) conduce a

$$
\begin{aligned}
R(t, T)= & r_{t} \frac{1-e^{-a(T-t)}}{a(T-t)}-\left(\frac{1-e^{-a(T-t)}}{a(T-t)}-1\right)\left(b-\frac{\sigma^{2}}{2 a^{2}}\right) \\
& +\frac{\sigma^{2}\left(1-e^{-a(T-t)}\right)^{2}}{4 a^{3}(T-t)} .
\end{aligned}
$$

Claramente $^{1}$, la tasa de interés de plazo más largo disponible en el mercado, también llamada tasa larga, $R(t, \infty)$, satisface

$$
R(t, \infty)=b-\frac{\sigma^{2}}{2 a^{2}},
$$

entonces se puede escribir como

$$
R(t, T)=R(t, \infty)+\left[r_{t}-R(t, \infty)\right] G(t, T)+\frac{\sigma^{2}(T-t)}{4 a} G^{2}(t, T),
$$

donde $G(t, T)=\frac{D(t, T)}{T-t}$. De esta manera, la curva de rendimiento, $R(t, T)$, es una función cuadrática de $G(t, T)$.

\footnotetext{
1 Se utiliza la regla de L'Hopital para el cálculo del límite.
} 
En la Gráfica 1 se muestra la curva de rendimiento en función de los parámetros $a$ y $b$. En este caso se tiene que $T-t=1, \sigma=0.001131 \mathrm{y}$ $r_{t}=0.0927$. Mientras que $a \in[0.01,0.5]$ y $b \in[0.01,0.5]$

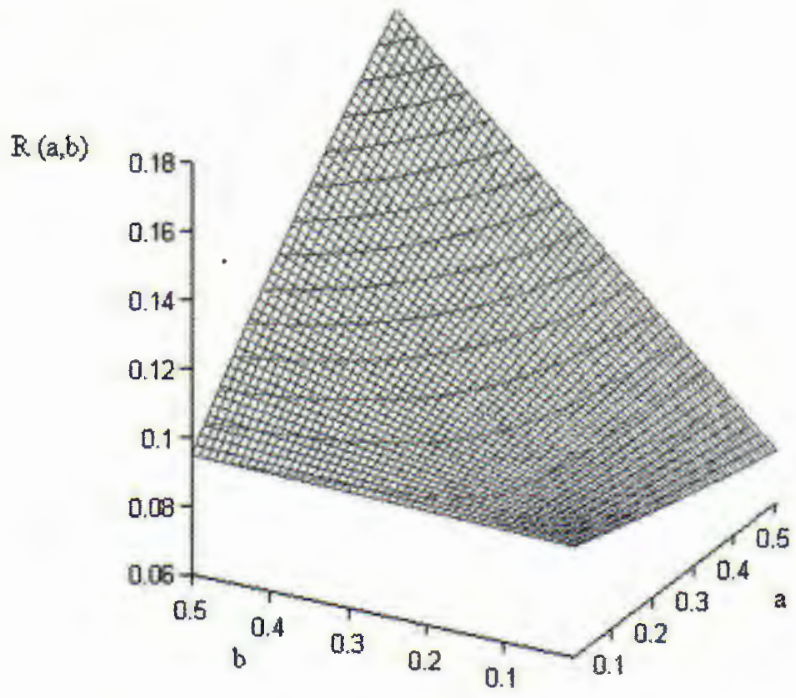

Gráfica 1. Curva de rendimiento en función de los parámetros $a$ y $b$.

\subsubsection{Precio de un bono cupón cero en el modelo de Vasicek}

A continuación se expresa el precio de un bono cupón cero en términos de la tasa larga, $R(t, \infty)$. Observe primero que

$$
B(t, T)=e^{-R(t, T)(T-t)} .
$$

Así, en virtud de la ecuación (12), el precio de un bono cupón cero, que se coloca en $t$ y que al vencimiento, $T$, paga una unidad monetaria, asociado al modelo de Vasicek de tasa corta se puede reescribir como:

$$
B(t, T)=\exp \left\{-R(t, \infty)(T-t)-\left[r_{t}-R(t, \infty)\right] D(t, T)-\frac{\sigma^{2}}{4 a} D^{2}(t, T)\right\} .
$$

En la Gráfica 2 se muestra la curva del precio de un bono cupon cero en función de los parámetros $a$ y $b$. En este caso se tiene que $T-t=1, \sigma=0.001131$ y $r_{t}=0.0927$. Mientras que $a \in[0.01,0.5]$ y $b \in[0.01,0.5]$ Observe que a medida que la velocidad conque el proceso se revierte a la media, $a$, aumenta el precio del bono, $B$, aumenta y se estabiliza en valores cercanos a su nominal. 


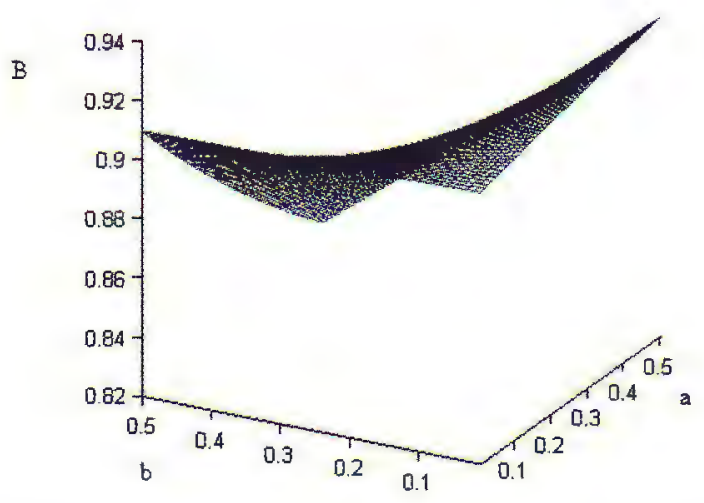

Gráfica 2. Precio de un bono cupón cero en función de los parámetros a y $b$.

\subsubsection{Tasa forward instantánea del modelo de Vasicek}

En esta sección se describe el comportamiento de la tasa forward asociada al modelo de Vasicek. La tasa forward instantánea está dada por la siguiente expresión:

$$
f(t, T)=-\frac{\partial}{\partial T} \ln B(t, T)=r_{t} \frac{\partial D}{\partial T}-\frac{\partial A}{\partial T} .
$$

Lo que conduce a

$$
f(t, T)=b-\left(b-r_{t}\right) e^{-a(T-t)}-\frac{\sigma^{2}}{2} D^{2}(t, T) .
$$

$Y$ es tal que $f(t, \infty) \equiv \lim _{T \rightarrow \infty} f(t, T)=b$.

A continuación se presenta la tasa forward instanánea del modelo de Vasicek en función de los parámetros $a$ y $b$.

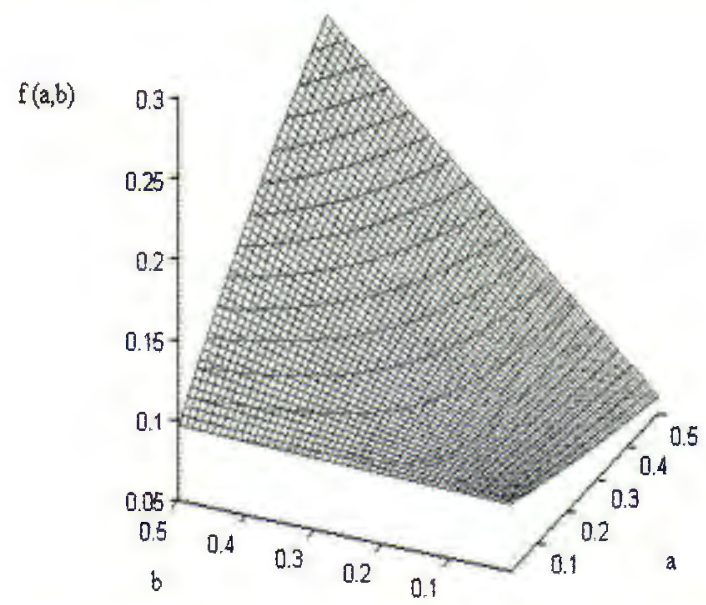

Gráfica 3. Tasa forward instantánea en función de los parámetros $a$ y $b$. 


\subsubsection{Aplicación del modelo de Vasicek}

En esta sección se lleva a cabo una aplicación del modelo de Vasicek. Asimismo, se muestra cómo los parámetros pueden ser estimados utilizando el modelo de regresión lineal simple con el supuesto estándar de errores normales no correlacionados, o bien con un proceso autoregresivo de orden uno. El modelo de Vasicek puede plantearse en términos discretos como una ecuación estocástica en diferencias. De la ecuación (1), se tiene

$$
r_{t}=a b+(1-a) r_{t-1}+\sigma \epsilon_{t},
$$

donde $\epsilon_{t} \sim \mathcal{N}(0,1)$. Si se escribe $\beta_{0}=a b$ y $\beta_{1}=1-a$, entonces

$$
r_{t}=\beta_{0}+\beta_{1} r_{t-1}+\varepsilon_{t}
$$

donde $\left\{\varepsilon_{t}\right\}$ son variables aleatorias independientes y normalmente distribuidas con media cero y varianza $\sigma^{2}, \varepsilon_{t} \sim \mathcal{N}\left(0, \sigma^{2}\right)$. Note que $\hat{a}=1-\hat{\beta}_{1}$ y $\hat{b}=\hat{\beta}_{0} / a$. La media (incondicional) de $r_{t}$ es

$$
\mathrm{E}\left[r_{t}\right]=\beta_{0} /\left(1-\beta_{1}\right)=b
$$

y su varianza (incondicional) está dada por

$$
\operatorname{Var}\left[r_{t}\right]=\sigma^{2} /\left(1-\beta_{1}\right)^{2}=\sigma^{2} /\left[1-(1-a)^{2}\right] .
$$

La varianza condicional de $r_{t}$, dado $r_{t-1}$ es, por supuesto $\sigma^{2}$.

\subsubsection{Comportamiento de la tasa de rendimiento, CETES 28}

A continuación se muestra la tasa anualizada, diaria, de CETES a 28 días para un periodo del 1 de noviembre de 2000 al 8 de marzo de 2006, sin impuestos ${ }^{2}$. Para este periodo el cambio porcentual de la tasa anualizada, diaria, de un día a otro llego a alcazar el $16.9 \%$ (sin impuesto).

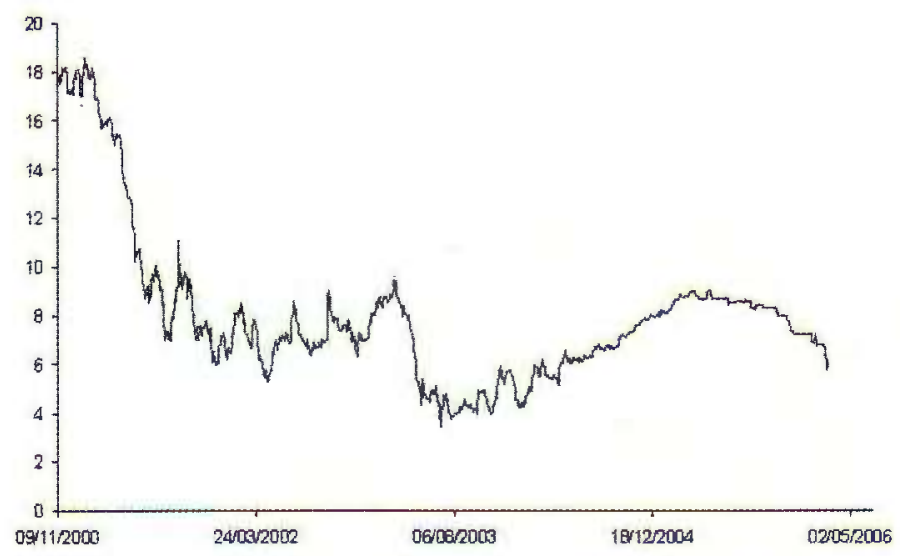

Gráfica 4. Tasa de rendimiento, cetes 28 , para el periodo 3 de mayo de 2004 al 8 de marzo de 2006 (sin impuesto).

2 Fuente: Valmer. 
Asimismo, se muestra la tasa anualizada de CETES A 28 días para un periodo del 3 de mayo de 2003 al 8 de marzo de 2006, (rendimiento anualizado de CETES a un día). En este periodo el cambio porcentual de la tasa anualizada, diaria, de un día a otro no supero el $5 \%$ (sin impuesto).

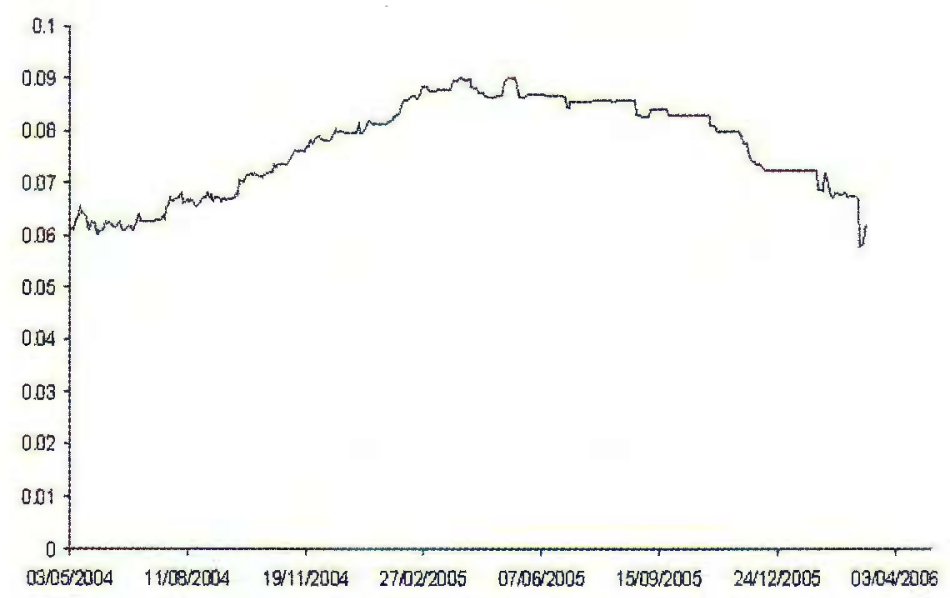

Gráfica 5. CETES A 28 dias para un periodo

del 3 de mayo de 2004 al 8 de marzo de 2006 (sin impuesto).

Los resultados de la estimación de los parámetros del modelo (16), con errores estándar entre paréntesis, son los siguientes:

$$
\begin{gathered}
r_{t}=0.000589+0.992968 r_{t-1} . \\
(0.000282) \quad(0.003580) .
\end{gathered}
$$

Los datos utilizados en la estimación corresponden a la tasa de rendimiento CETES 28 para el periodo del 3 de mayo de 2004 al 8 de marzo de 2006, sin impuesto.

Note que, $\mathrm{E}\left[\beta_{0}\right]=\hat{\beta}_{0}, \mathrm{E}\left[\beta_{1}\right]=\hat{\beta}_{1}$ y $\mathrm{E}\left[\epsilon_{t}\right]=0$. En este caso, se puede apreciar que las estimaciones son significativamente distintas de cero con un $95 \%$ de confianza. La gráfica 6 muestra la estructura de plazos definida a través de la ecuación (10) con $a=0.007032, b=0.083772, r_{t}=0.0621$ y $\sigma=0.090579173$. Como puede observarse, la estructura de plazos es positiva, creciente y en el largo plazo, se estabiliza en un valor cercano al $8.38 \%$.

Se sabe que $R(t, T)$ puede tener cualquier comportamiento: cóncava, convexa, etc. y que $R(t, T)$ puede ser negativa para algunos valores de los parámetros.

\subsection{Modelo de la tasa corta de Cox, Ingersoll y Ross, CIR}

A continuación se presentan algunas características del modelo CIR, en particular su función de densidad ji-cuadrada, la curva de rendimiento, la estructura de plazos para un cierto conjunto de datos 


\subsubsection{Características y extensiones del modelo CIR}

El modelo Cox, Ingersoll y Ross (1985b) es un modelo de valuación de activos en equilibrio para la estructura de plazos de tasas de interés. El modelo proporciona soluciones para la valuación de bonos y caracteriza completamente la estructura de plazos la cual incorpora la prima al riesgo y la esperanza de futuras tasas de interés. Este modelo ha sido frecuentemente presentado como un modelo de un sólo factor, más sin embargo, CIR mostraron como incorporar factores múltiples así como la extensión del modelo a la valuación de bonos nominales y productos derivados. El modelo CIR ha resultado ser un modelo de gran trascendencia en la ciencias económicas financieras por varias razones, en las que podemos destacar las siguientes: proporciona una fuerte conexión entre la teoría de valuación de activos de manera intertemporal y la estructura de plazos de tasas de interés, preserva el requerimiento de que las tasas de interés sean no negativas, y proporciona soluciones cerradas relativamente simples en la valuación de bonos. Asimismo, el modelo es una herramienta útil para la valuación de productos derivados de tasas de interés. Cabe señalar que se han llevado a cabo investigaciones para extender el modelo CIR a varios factores en la cual la estimación de las variables de estado no observables son generadas por un filtro de Kalman. En particular se han realizado estimaciones del modelo CIR con tres factores y varias pruebas para determinar si el modelo captura completamente la variabilidad de la estructura de plazos de tasas de interés en el tiempo. El modelo de tres factores es una extensión del modelo de dos factores desarrollado por Longstaff y Schwartz (1992), en el cual los dos factores utilizados son la tasa corta y la volatilidad de la tasa de interés siendo ésta un análisis alternativo al de Brennan y Schwartz (1979) en la cual los dos factores de riesgo son la tasa corta y la tasa de largo plazo. Por lo que si la tasa corta, la tasa de largo plazo y la volatilidad de la tasa corta impactan en la estrucutra de plazos de tasas de interés entonces da lugar a un modelo de tres factores. Es importante destacar que el modelo CIR es no lineal por lo que el modelo tiene asociado una distribución ji-cuadrada no central la cual se explicara con mayor detenimiento en posteriores secciones.

\subsubsection{Fundamentos del modelo CIR}

Es imprescindible mencionar que en la estructura de plazos generada con el modelo de Vasicek, el cual se obtiene con $\beta=0$, puede producir tasas negativas, con probabilidad positiva. Afortunadamente, esta limitación desaparece en el modelo propuesto por Cox, Ingersoll y Ross (CIR), en 1985, en su artículo "A Theory of the Term Structure of Interest Rates" publicado en Econometrica, ya que en su modelo las tasas siempre son positivas. Esta sección se concentra en una dinámica de la tasa corta conducida por la siguiente ecuación diferencial estocástica:

$$
\mathrm{d} r_{t}=a\left(b-r_{t}\right) \mathrm{d} t+\sigma \sqrt{r_{t}} \mathrm{~d} W_{t} .
$$

Es importante observar que al considerar $\sqrt{r_{t}}$ en el término estocástico el proceso de la tasa corta deja de ser Normal. De hecho, en este caso, la distribución de la tasa corta corresponde a una $\chi^{2}$ no central. Este proceso presenta reversión a la media como en el modelo de Vasicek, pero la varianza es proporcional a $\sigma^{2} r_{t}$ por unidad de tiempo. Esto significa que conforme la tasa de interés corta aumenta, la desviación estándar aumenta. 


\subsubsection{Ecuación diferencial parcial del comportamiento del precio de un bono cupón cero en el modelo de CIR}

En un mundo neutral al riesgo, el precio del bono cupón cero, denotado por $B(t, T)$, satisface la ecuación diferencial parcial parabólica dada por

$$
\frac{\partial B}{\partial t}+\frac{1}{2} \sigma^{2} r_{t} \frac{\partial^{2} B}{\partial r_{t}^{2}}+a\left(b-r_{t}\right) \frac{\partial B}{\partial r_{t}}-r B_{t}=0
$$

junto con la condición de frontera $B(T, T)=1$.

\subsubsection{Solución de la ecuación diferencial parcial del precio de un bono cupón cero}

En esta sección se presenta la solución de la ecuación diferencial parcial que caracteriza el precio de un bono cupón cero en el modelo CIR. Dado que la ecuación diferencial parcial parabólica no presenta derivadas parciales cruzadas, se supone una solución en variables separables, es decir :

$$
B(t, T)=e^{A(t, T)-r_{t} D(t, T)} .
$$

Claramente, $A(T, T)=D(T, T)=0$ ya que el valor nominal del bono está dado por

$$
B(T, T)=e^{A(T, T)-r_{T} D(T, T)}=1 .
$$

En este caso, se obtiene de manera simple que

$$
D(t, T)=\frac{2\left(e^{\sqrt{a^{2}+2 \sigma^{2}}(T-t)}-1\right)}{\left(a+\sqrt{a^{2}+2 \sigma^{2}}\right)\left(e^{\sqrt{a^{2}+2 \sigma^{2}}(T-t)}-1\right)+2 \sqrt{a^{2}+2 \sigma^{2}}} .
$$

y

$$
A(t, T)=\ln \left[\frac{2 \sqrt{a^{2}+2 \sigma^{2}} e^{\left(a+\sqrt{a^{2}+2 \sigma^{2}}\right)(T-t) / 2}}{\left(a+\sqrt{a^{2}+2 \sigma^{2}}\right)\left(e^{\sqrt{a^{2}+2 \sigma^{2}}(T-t)}-1\right)+2 \sqrt{a^{2}+2 \sigma^{2}}}\right]^{2 a b / \sigma^{2}} .
$$

\subsubsection{Curva de rendimiento del modelo CIR}

En el caso del modelo CIR es posible obtener curvas de rendimiento con pendiente positiva, con pendiente negativa o con jorobas. La curva de rendimiento del modelo CIR se calcula como sigue:

$$
\begin{aligned}
R(t, T) & =-\frac{\ln B(t, T)}{T-t} \\
& =\frac{r_{t} D(t, T)-A(t, T)}{T-t},
\end{aligned}
$$

la cual es una función lineal de $r_{t}$. En este caso se puede demostrar que

$$
R(t, \infty) \equiv \lim _{T \rightarrow \infty} R(t, T)=\frac{2 a b}{a+\sqrt{a^{2}+2 \sigma^{2}}} .
$$




\subsubsection{Función de densidad del modelo CIR}

El modelo CIR ha sido muy utilizado en la valuación de productos derivados de tasas de intéres. Como se menciono la tasa spot es no negativa. Se sabe que la tasa spot es no negativa cuando $\sigma^{2} \leq 2 \alpha$, mientras que $r(t) \geq 0$ cuando $\sigma^{2}>2 \alpha$. La función de densidad del modelo CIR está dada por

$$
f\left(r_{t}\right)=c e^{-c\left(r_{t}+v\right)}\left(\frac{r_{t}}{v}\right)^{q / 2} I_{q}\left(2 c \sqrt{r_{t} v}\right) ; \quad r_{t} \geq 0,
$$

donde

$$
\begin{aligned}
& c=\frac{2 a}{\sigma^{2}\left(1-e^{-a t}\right)}, \quad v=r_{0} e^{-a t}, \quad r(0)=r_{0} \\
& q=\frac{2 a b}{\sigma^{2}}-1
\end{aligned}
$$

y en la que la función

$$
I_{q}(x)=\sum_{k=0}^{\infty} \frac{\left(\frac{x}{2}\right)^{2 k+q}}{k ! \Gamma(k+q+1)}
$$

es la función modificada de Besse ${ }^{3}$ de primera clase y de orden $q$. Mientras que $\Gamma(x)$ es la función gamma. La función de densidad es una función de distribución ji-cuadrada no central, con $2(q+1)$ grados de libertad y no centralidad $2 c v$. Es decir,

$$
\begin{aligned}
2(q+1) & =2\left(\frac{2 a b}{\sigma^{2}}-1+1\right) \\
& =\frac{4 a b}{\sigma^{2}}
\end{aligned}
$$

y no centralidad

$$
\begin{aligned}
2 c v & =2\left(\frac{2 a}{\sigma^{2}\left(1-e^{-a t}\right)}\right) r_{0} e^{-a t} \\
& =\left(\frac{4 a r_{0} e^{-a t}}{\sigma^{2}\left(1-e^{-a t}\right)}\right) .
\end{aligned}
$$

3 La función modificada de Bessel de primera clase y de orden $q$ está dada por

$$
I_{q}(x)=\sum_{k=0}^{\infty} \frac{\left(\frac{x}{2}\right)^{2 k+q}}{k !(k+q) !} .
$$

Se tiene que $\Gamma(\alpha)=(\alpha-1)$ !. Sea $\alpha=k+q+1$, entonces $\Gamma(\alpha)=\Gamma(k+q+1)=$ $(k+q+1-1) !=(k+q)$ !. Por lo tanto, la función modificada de Bessel de primera clase y de orden $q$ se puede escribir como

$$
I_{q}(x)=\sum_{k=0}^{\infty} \frac{\left(\frac{x}{2}\right)^{2 k+q}}{k ! \Gamma(k+q+1)} .
$$


La función de densidad $f\left(r_{t}\right)$ se puede escribir en forma explicita en términos de la función de distribución Poisson y la función gamma. Para ello, se considera la función modificada de Bessel de primera clase y de orden $q$ en la que se efectua el cambio de variable dado por $x=2 c \sqrt{r_{t} v}$. Esto es,

$$
I_{q}(x)=I_{q}\left(2 c \sqrt{r_{t} v}\right)=\sum_{k=0}^{\infty} \frac{1}{k ! \Gamma(k+q+1)}\left(c \sqrt{r_{t} \bar{v}}\right)^{2 k+q} .
$$

Si se sustituye la función anterior en la función de densidad, (24), se tiene

$$
f\left(r_{t}\right)=\sum_{k=0}^{\infty} \frac{(c v)^{k}}{k !} e^{-c v} \frac{1}{\Gamma(k+q+1)} c^{q+k+1} r_{t}^{q+k} e^{-c r_{t}} .
$$

Sea $\alpha=k+q+1$ y $c=\lambda$, entonces la ecuación anterior se puede escribir como

$$
f\left(r_{t}\right)=\sum_{k=0}^{\infty} \frac{(\lambda v)^{k}}{k !} e^{-\lambda v} \frac{1}{\Gamma(\alpha)} \lambda^{\alpha} r_{t}^{\alpha-1} e^{-\lambda r_{t}}
$$

que representa la función de densidad del modelo CIR en términos de la función de densidad gamma y Poisson. Como se puede observar es el producto de una función de densidad gamma con parámetro de forma $\theta=1 / \lambda>0$ y $\alpha>0$, y la función Poisson con parámetro $\lambda$. Si se considera que

$$
h_{\alpha, \lambda}\left(r_{t}\right)=h_{k+q+1_{q} c}\left(r_{t}\right)=\frac{1}{\Gamma(\alpha)} \lambda^{\alpha} r_{t}^{\alpha-1} e^{-\lambda r_{t}},
$$

ésta es la función de densidad de la distribución gamma con media $\mathrm{E}\left[r_{t}\right]=\alpha / \lambda$ y varianza $\operatorname{Var}\left(r_{t}\right)=\alpha / \lambda^{2}$.

\subsubsection{Función de distribución acumulada del modelo CIR}

La probabilidad de que una variable aleatoria ji-cuadrada, $\chi^{2}$, no central sea menor o igual a un cierto valor $\chi_{\nu, \delta}^{2} \operatorname{con} \nu$ grados de libertad y parámetro de no centralidad $\delta$, está dada por la función de distribución acumulada

$$
\begin{aligned}
F\left(r_{t} ; \nu, \delta\right) & =\mathrm{P}_{\text {rob }}\left\{\mathcal{X}_{\nu, \delta}^{2} \leq \chi_{\nu, \delta}^{2}\right\} \\
& =\sum_{k=0}^{\infty} \frac{(\lambda \nu)^{k}}{k !} e^{-\lambda v} \mathrm{P}_{\text {rob }}\left\{\mathcal{X}^{2} \leq \chi_{\nu, \delta}^{2}\right\} .
\end{aligned}
$$

\subsection{Inversa de la función de distribución acumulada de una ji-cua- drada no central, CIR}

La inversa de la función de distribución acumulada de una ji-cuadrada no central con probabilidad $p, \nu$ los grados de libertad y $\delta$ el parámetro de no centralidad está dada por

$$
z=F^{-1}(p ; \nu, \delta=2 c v)=\left\{z: F\left(r_{t} ; \nu, \delta=2 c v\right)=p\right\}
$$


donde

$$
F\left(r_{t} ; \nu=2(q+1), \delta=2 c v\right)=\sum_{k=0}^{\infty} \frac{(\lambda v)^{k}}{k !} e^{-\lambda v} \mathrm{P}_{r o b}\left\{\mathcal{X}_{\nu=2(q+1)}^{2} \leq \chi_{\nu, \delta}^{2}\right\}
$$

\subsection{Distribución de la tasa corta en el modelo CIR}

El objetivo de esta sección es determinar la distribución de la tasa corta en el modelo CIR. Considere el proceso de Orstein-Uhlenbeck

$$
\mathrm{d} y_{t}=-a y_{t} \mathrm{~d} t+\sigma \mathrm{d} U_{t}
$$

donde $a, \sigma>0$ y $\left\{U_{t}\right\}_{t>0}$ es un movimiento Browniano definido sobre un espacio fijo de probabilidad $(\Omega, \mathcal{F}, \mathbb{P})$. La solución de este proceso es similar a la de una ecuación diferencial no homogénea de primer orden, esto es

$$
y_{t}=y_{0} e^{-a t}+\sigma \int_{0}^{t} e^{-a(t-s)} \mathrm{d} U_{s}
$$

Así, $y_{t}$ es normal con media condicional $\mathrm{E}\left[y_{t} \mid y_{0}\right]=y_{0} e^{-a t}$ y varianza condicional dada por $\operatorname{Var}\left[y_{t} \mid y_{0}\right]=\sigma^{2} \int_{0}^{t} e^{-2 a(t-s)} \mathrm{d} s=\frac{\sigma^{2}}{2 a}\left(1-e^{-2 a t}\right)$. Observe también que una aplicación del lema de Itô a $x_{t}=y_{t}^{2}$ conduce a

$$
\mathrm{d} x_{t}=2 y_{t} \mathrm{~d} y+\sigma^{2} \mathrm{~d} t
$$

Suponga ahora que se tienen $U_{1 t}, U_{2 t}, \ldots, U_{n t}$ movimientos Brownianos independientes definidos sobre $(\Omega, \mathcal{F}, \mathbb{P})$ y defina los siguientes $n$ procesos de OrsteinUhlenbeck:

$$
\mathrm{d} y_{k t}=-\frac{1}{2} a y_{k t} \mathrm{~d} t+\frac{1}{2} \sigma \mathrm{d} U_{k t}, \ldots \quad k=1,2, \ldots, n .
$$

En cuyo caso, se tiene que $y_{k t}=e^{-a t / 2}\left(y_{k 0}+\frac{1}{2} \sigma \int_{0}^{t} e^{a s / 2} \mathrm{~d} U_{k s}\right), \quad k=$ $1,2, \ldots, n$. y es tal que $\mathrm{E}\left[y_{k t} \mid y_{k 0}\right]=y_{k 0} e^{-a t / 2} \equiv \mu_{k, t} \mathrm{y}$

$$
\operatorname{Var}\left[y_{k t} \mid y_{k 0}\right]=\frac{\sigma^{2}}{4} \int_{0}^{t} e^{-a(t-s)} \mathrm{d} s=\frac{\sigma^{2}}{4 a}\left(1-e^{-a t}\right) \equiv \nu_{t}^{2}
$$

Considere ahora el proceso

$$
\begin{aligned}
r_{t} & =y_{1 t}^{2}+y_{2 t}^{2}+\ldots+y_{n t}^{2} \\
& =\sum_{k=1}^{n} y_{k t}^{2}
\end{aligned}
$$

entonces, $\frac{r_{t}}{\nu_{t}^{2}} \sim \chi^{2}\left(n, \delta_{t}\right)$ con parámetro de no centralidad $\delta_{t}=\frac{1}{\nu_{t}^{2}} \sum_{k=1}^{n} \mu_{k, t}^{2}$. Es decir, $r_{t} / \nu_{t}^{2}$ sigue una distribución ji cuadrada no central con $n$ grados de 
libertad y parámetro de no centralidad $\delta_{t}$. Es importante observar también, que

$$
\mathrm{E}\left[\frac{r_{t}}{\nu_{t}^{2}} \mid r_{0}\right]=\frac{1}{\nu_{t}^{2}} \sum_{k=1}^{n} \mathrm{E}\left[y_{k t}^{2} \mid y_{k 0}\right]=n+\frac{e^{-a t}}{\nu_{t}^{2}} r_{0}
$$

Por lo tanto, de (39), se sigue que

$$
\operatorname{Var}\left[\frac{r_{t}}{\nu_{t}^{2}}\right]=\frac{r_{0}^{2} e^{-a t}}{\nu_{t}^{4}}+\frac{n}{\nu_{t}^{2}}-\left(n+\frac{e^{-a t}}{\nu_{t}^{2}} r_{0}\right)^{2}
$$

2.4.1 Correspondencia entre los parámetros de la distribución ji-cuadrada y el modelo CIR

Por otro lado, a partir de (36), se sigue que

$$
\begin{aligned}
\mathrm{d} r_{t} & =\sum_{k=1}^{n} \mathrm{~d} y_{k t}^{2} \\
& =\left(\frac{n \sigma^{2}}{4}-a r_{t}\right) \mathrm{d} t+\sigma \sqrt{r_{t}} \sum_{k=1}^{n} \frac{y_{k t} \mathrm{~d} U_{k t}}{\sqrt{r_{t}}}
\end{aligned}
$$

Se define

$$
\mathrm{d} W_{t} \equiv \sum_{k=1}^{n} \frac{y_{k t} \mathrm{~d} U_{k t}}{\sqrt{r_{t}}}
$$

Claramente $W_{t}$ es una martingala, pues no tiene tendencia. Si se define $V_{t}=$ $W_{t}^{2}$, se tiene que

$$
\begin{aligned}
\mathrm{d} V_{t} & =\frac{1}{2} \frac{\partial^{2} V_{t}}{\partial W_{t}^{2}} \sum_{k=1}^{n} \frac{y_{k t}^{2}}{r_{t}} \mathrm{~d} t+\frac{\partial V_{t}}{\partial W_{t}} \sum_{k=1}^{n} \frac{y_{k t} \mathrm{~d} W_{k t}}{\sqrt{r_{t}}} \\
& =\frac{1}{r_{t}} \sum_{k=1}^{n} y_{k t}^{2} \mathrm{~d} t+2 W_{t} \mathrm{~d} W_{t} \\
& =\mathrm{d} t+2 W_{t} \mathrm{~d} W_{t} .
\end{aligned}
$$

Por lo tanto,

$$
W_{t}^{2}-t=2 \int_{0}^{t} W_{s} \mathrm{~d} W_{s}
$$

es una martingala. En virtud del teorema de representación de Lévy, se tiene que $W_{t}$ es un movimiento Browniano definido en $(\Omega, \mathcal{F}, \mathbb{P})$. En consecuencia, se puede escribir

$$
\mathrm{d} r_{t}=\left(\frac{n \sigma^{2}}{4}-a r_{t}\right) \mathrm{d} t+\sigma \sqrt{r_{t}} \mathrm{~d} W_{t}
$$


Si se compara (45) con (18) se tiene que $a b=n \sigma^{2} / 4$ Por lo tanto, al despejar $n$ de la expresión anterior se tiene

$$
n=\frac{4 a b}{\sigma^{2}} \text {. }
$$

La ecuación anterior tiene aún sentido si $n$ no es un entero positivo. Por lo que, el parámetro de no centralidad satisface

$$
\begin{aligned}
\delta_{t} & =\frac{1}{\nu_{t}^{2}} \sum_{k=1}^{n} y_{k 0}^{2} e^{-a t / 2} \\
& =\frac{1}{\nu_{t}^{2}} r_{0} e^{-a t} .
\end{aligned}
$$

De acuerdo con (1), se puede concluir que

$$
\frac{4 a}{\sigma^{2}\left(1-e^{-a t}\right)} r_{t} \sim \chi^{2}\left(\frac{4 a b}{\sigma^{2}}, \frac{4 a r_{0} e^{-a t}}{\sigma^{2}\left(1-e^{-a t}\right)}\right) .
$$

Note que, $2 c r_{t}=\frac{4 a}{\sigma^{2}\left(1-e^{-a t}\right)} r_{t}$.

\subsubsection{Cambio de variable en el modelo CIR para obtener volatilidad constante}

Se considera un cambio de variable que permite reescribir la dinámica de la tasa corta CIR en una ecuación diferencial estocástica en la que la varianza o volatilidad es una constante. Para ello, si se define $y_{t} \equiv y\left(r_{t}, t\right)=2 \sqrt{r_{t}}$, se tiene que al aplicar el lema de Itô, conduce a la ecuación diferencial d $y_{t}$ donde $y_{t}$ es función de la tasa corta, esto es

$$
\mathrm{d} y_{t}=\left[\left(2 a b-\frac{\sigma^{2}}{2}\right) \frac{1}{y_{t}}-\frac{a}{2} y_{t}\right] \mathrm{d} t+\sigma \mathrm{d} W_{t}
$$

donde $\sigma$, es constante. Los parámetros se pueden estimar por mínimos cuadrados ordinarios como se presenta más adelante.

\subsubsection{Estimación de los parámetros}

A continuación se presentan varios métodos de estimación de los parámetros del modelo CIR, los cuales utilizan un registro histórico de la tasa corta o de la curva de rendimiento.

\subsubsection{Estimación de parámetros en el modelo CIR con MGM}

En esta sección se discute sobre la estimación de parámetros en el modelo CIR mediante el método generalizado de momentos (MGM). Considere la versión discreta del modelo CIR

$$
r_{t+1}=\beta_{0}+\beta_{1} r_{t}+\sqrt{r_{t}} \epsilon_{t}
$$


Tabla 2.

\begin{tabular}{|c|c|c|c|c|}
\hline \multicolumn{5}{|c|}{ Estimación de parámetros } \\
\hline Periodo & $a$ & $b$ & $\sigma^{2}$ & $\sigma$ \\
\hline $\begin{array}{c}03 / \text { mayo/2004- } \\
08 / 03 / 2006(\mathrm{MCO})\end{array}$ & 0.005900 & 0.088000 & 0.0058138 & 0.076242 \\
\hline MGM: & 0.005819 & 0.088002 & 0.0057141 & 0.075592 \\
\hline
\end{tabular}

Los resultados de la estimación de los parámetros del modelo (49), con errores estándar entre paréntesis, son los siguientes:

$$
\begin{gathered}
y_{t+1}=\beta_{1} y_{t}^{-1}+\beta_{2} y_{t}+\epsilon_{t}, \\
y_{t+1}=0.0059 y_{t}^{-1}+0.088 y_{t}+0.007624 \varepsilon_{t} . \\
(0.001102) \quad(0.011096) .
\end{gathered}
$$

Note que, $\mathrm{E}\left[\beta_{0}\right]=\hat{\beta}_{0}, \mathrm{E}\left[\beta_{1}\right]=\hat{\beta}_{1}$ y $\mathrm{E}\left[\epsilon_{t}\right]=0$. En el caso del modelo CIR es posible obtener curvas de rendimiento con pendiente positiva, con pendiente negativa o con jorobas. En este caso, la curva de rendimiento presenta una pendiente positiva como se puede apreciar en la siguiente gráfica.

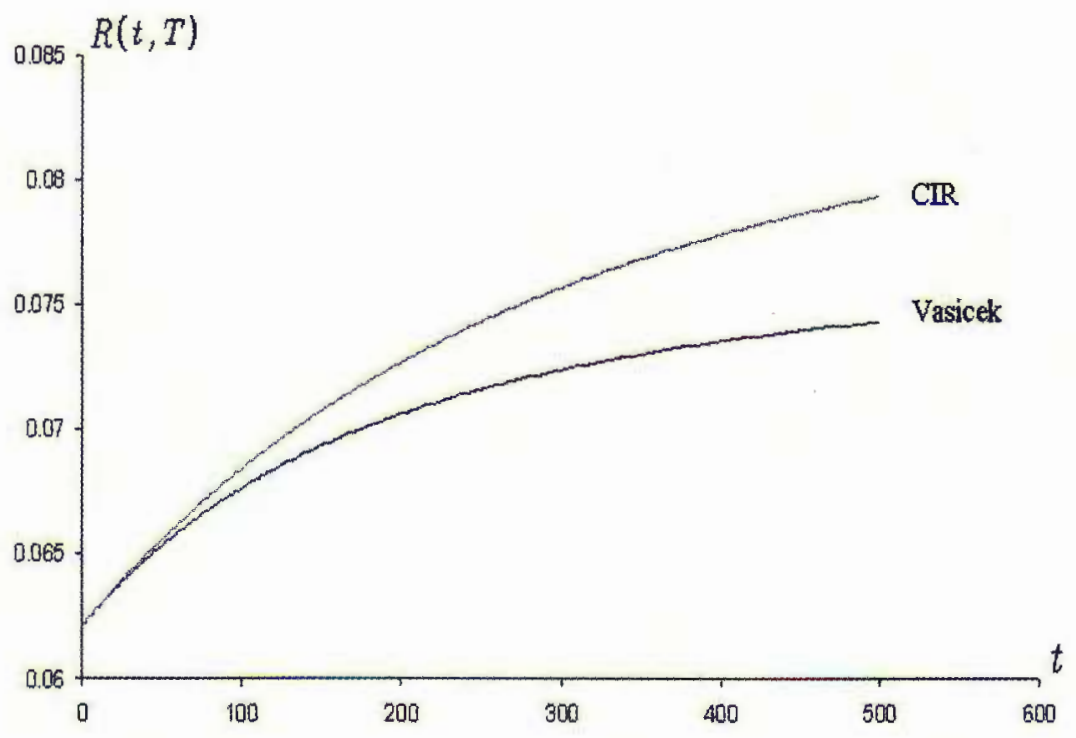

Gráfica 6. Estructura de plazos estimada (CIR) y Vasicek, R(t,T). 


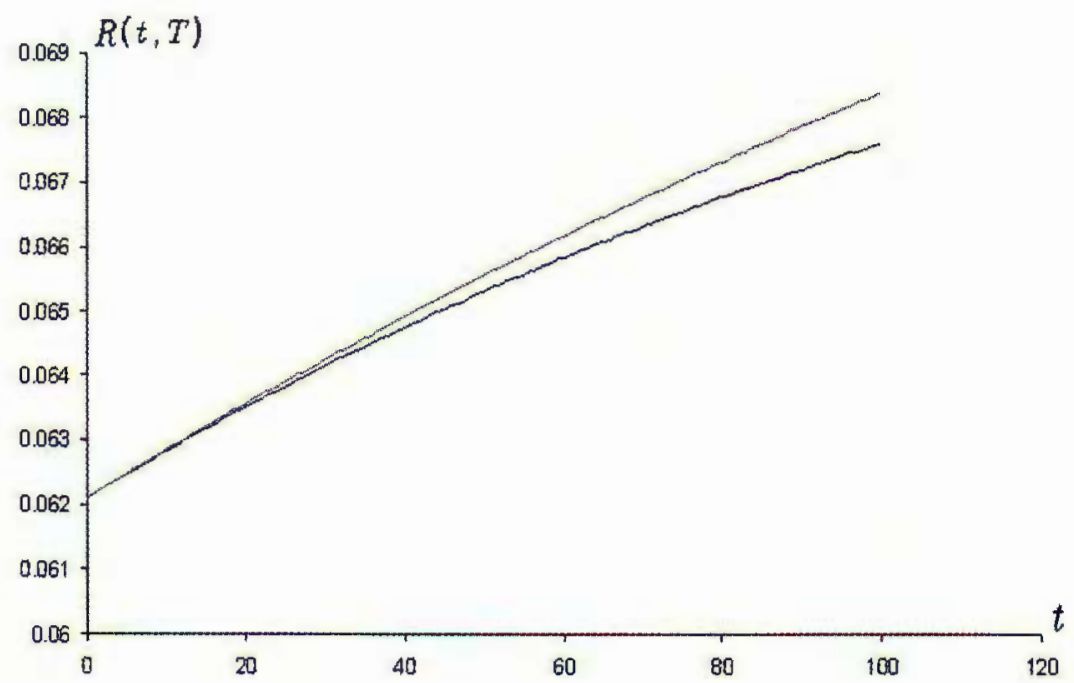

Gráfica 7. $R(t, T)$. Estructura de plazos estimada (CIR) y Vasicek.

En la gráfica 7 se comparan la estructura de plazos del modelo de Vasicek y del modelo de Cox-Ingersoll-Ross. En la que se aprecia que la estrucutura de plazos del modelo CIR está por arriba de Vasicek. En la gráfica 7 en la que se tiene plazos de hasta un año, la estructura de plazos alcanza un valor de hasta $6.216 \%$. Mientras que a largo plazo, la tasa es de $6.78 \%$.

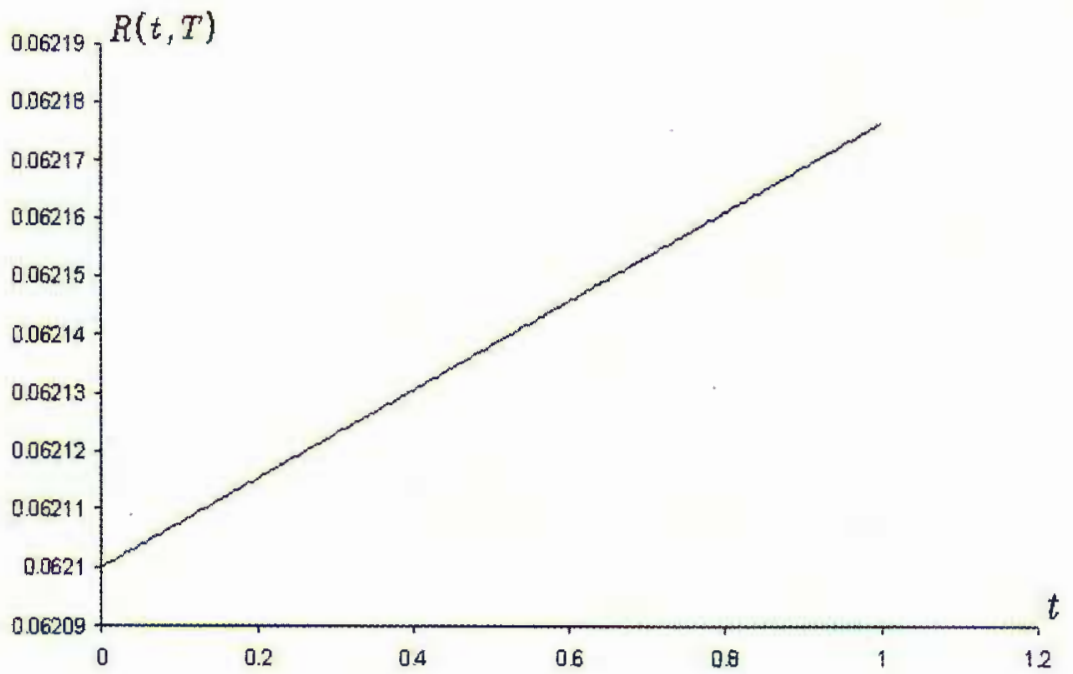

Gráfica 8. $R(t, T)$ con $t$ en dias. Estructura de plazos estimada (CIR). 


\subsubsection{Estimación de los parámetros del modelo de Cox, Ingersoll y Ross con el MGM}

En esta sección se lleva a cabo una aplicación del modelo CIR utilizando MGM para la estimación de los parámetros. Para fines prácticos, el modelo CIR puede plantearse en términos discretos como una ecuación estocástica en diferencias:

$$
r_{t+1}=\beta_{0}+\beta_{1} r_{t}+\sqrt{r_{t}} \epsilon_{t},
$$

donde $\beta_{0}=a b$ y $\beta_{1}=1-a$, junto con las restricciones:

$$
\sum_{t=1}^{n} \epsilon_{t}=0, \quad \sum_{t=1}^{n} \epsilon_{t} \epsilon_{t-1}=0 \quad \text { y } \quad \sum_{t=1}^{n}\left(\epsilon_{t}^{2}-r_{t} \sigma^{2}\right)=0 .
$$

\subsection{Función de densidad y función de distribución acumulada para CETES 28}

Acontinuación se muestra la función de densidad ji-cuadrada no central y la función de distribución acumulada del comportamiento de la tasa corta de CETES 28 , diaria, del periodo 3 de mayo de 2004 al 8 de marzo de 2006. La función utilizada, de densidad del modelo CIR, puede verse en la ecuación (29), donde los valores de los parámetros de $a, b$ y $\sigma$ aparecen en la Tabla 2.

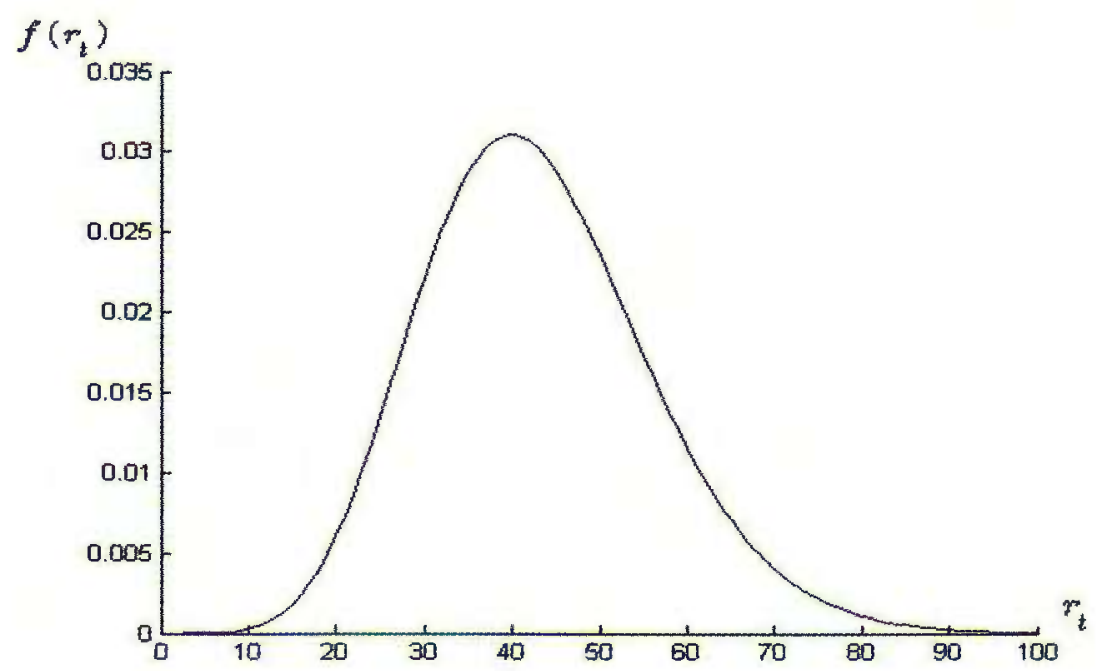

Gráfica 9. Función de densidad de probabilidad ji-cuadrada no central con $\nu=0.357277983$ y $\delta=42.60704074$. 
De esta manera la función de densidad del modelo CIR con $\nu=0.357277983$ y $\delta=42.60704074$ se presenta en la gráfica 9. Asimismo, la inversa de la función de distribución acumulada de una ji-cuadrada no central, tasa corta, con probabilidad $p=0.05, \nu=0.357277983$ grados de libertad y $\delta=42.60704074$ parámetro de no centralidad, es decir

$$
r_{t}=z=F^{-1}(p ; \nu, \delta=2 c v)=\left\{z: F\left(r_{t} ; \nu, \delta=2 c v\right)=p\right\}
$$

está en la gráfica 10.

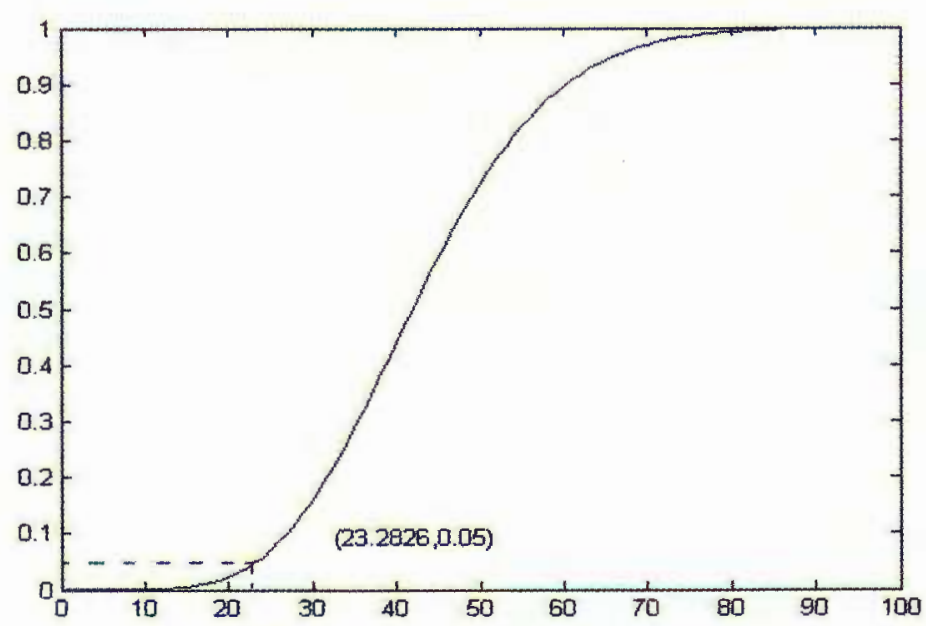

Gráfica 10. Función de de distribución acumulada ji-cuadrada no central $\operatorname{con} \nu=0.357277983$ y $\delta=42.60704074$.

\section{Valor en riesgo}

Con el objeto de calcular el valor en riesgo, también llamada el VaR, de bonos cupon cero, a continuación se presenta una introducción al valor en riesgo, el concepto de valor en riesgo así como el valor en riesgo del modelo de Vasicek y que permitira entender mejor la aplicación de este importante concepto en el mercado financiero mexicano.

El valor en riesgo es una de las medidas que se utilizan con mayor frecuencia, por los intermediarios financieros, en la estimación de pérdidas potenciales, ya sea en el valor o en el rendimiento de un portafolio debido a movimientos en los factores de riesgo mercado, en un periodo de tiempo y con un determinado nivel de confianza dado. 


\subsection{El concepto de valor en riesgo, paramétrico}

En esta sección se presenta la definición formal del valor en riesgo parámetrico del cambio en el valor de un portafolio.

El valor en riesgo de $X$ al nivel $1-q$ denotado por $-\mathrm{VaR}_{1-q}^{X}$, se define como el peor valor del portafolio, en un periodo de tiempo dado, $[t, T]$, para un intervalo de confianza del $(1-q) 100 \%$. Esto es,

$$
\mathbb{P}_{\theta}\left\{-\operatorname{VaR}_{1-q}^{X} \leq X\right\}=1-q .
$$

Entonces,

$$
\operatorname{VaR}_{1-q}^{X}=-\inf \left\{x \in \mathbb{R} \mid \mathbb{P}_{\theta}\{X>x\} \leq 1-q\right\} .
$$

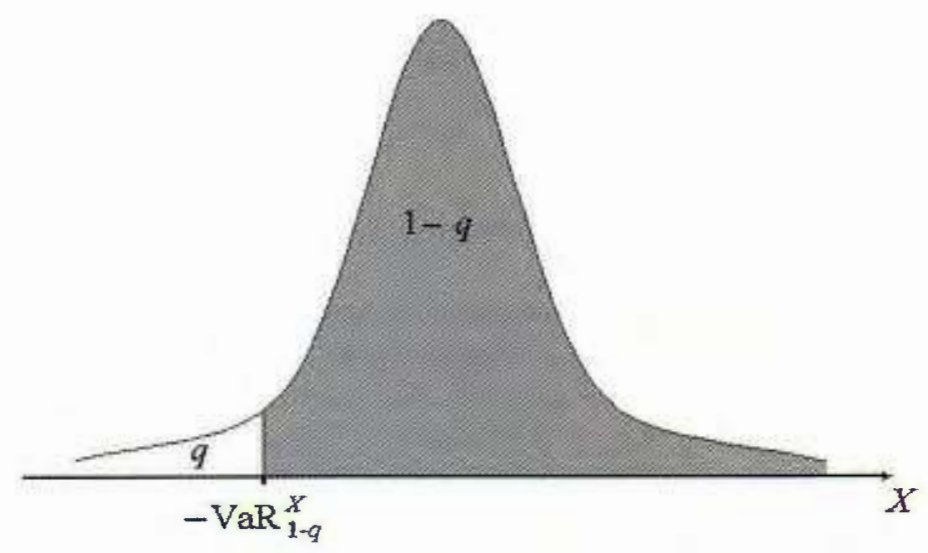

Figura 1. Valor en riesgo de $X$ al nivel $1-q$.

En la figura 1, se ilustra el concepto del valor en riesgo, es decir el número $\operatorname{VaR}_{1-q}^{X}$ es una estimación estadística del peor valor de $X$ con cierto grado de confianza en un intervalo de tiempo dado. Si se considera un portafolio de inversión, en el mercado financiero, el $\operatorname{VaR}_{1-q}^{X}$ representa la máxima cantidad de dinero que se puede perder dentro de un cierto periodo de tiempo.

\subsection{Valor en riesgo bajo el supuesto de normalidad}

Posiblemente, el supuesto normalidad en el VaR ha contribuido de manera muy importante a que el mismo VaR sea tan popular. Bajo este supuesto, el cálculo del VaR se convierte en una expresión fácil de calcular.

Si el cambio de valor en un portafolio durante $[t, T], X$, es una variable aleatoria continua y $F$ es su función de distribución, entonces $\operatorname{VaR}_{1-q}^{X}=F^{-1}(q)$, es decir, $\operatorname{VaR}_{1-q}^{X}$ es el quantil $q$ de $F$. Por ejemplo, si el cambio en el valor de $\Pi_{t}$ satisface

$$
\mathrm{d} \Pi_{t}=\mu \mathrm{d} t+\sigma \mathrm{d} W_{t},
$$


donde $\mu \in \mathbb{R}, \sigma>0 \mathrm{y}\left(W_{t}\right)_{t \in[0, T]}$ es un movimiento Browniano definido en un espacio de probabilidad equipado con su filtración aumentada,

$$
\left(\Omega, \mathcal{F},\left(\mathcal{F}_{t}\right)_{t \in[0, T]}, \mathbb{P}\right),
$$

entonces

$$
X=\Pi_{T}-\Pi_{t} \sim \mathcal{N}\left(\mu(T-t), \sigma^{2}(T-t)\right) .
$$

Por lo que,

$$
\begin{aligned}
\operatorname{VaR}_{1-q}^{X} & =z_{q} \sigma \sqrt{T-t}+\mathrm{E}^{\mathbb{P}}\left[-X \mid \mathcal{F}_{t}\right] \\
& =z_{q} \sigma \sqrt{T-t}-\mu(T-t) .
\end{aligned}
$$

A partir de las tablas de quantiles de la función de distribución acumulada de una variable normal estándar, se tiene que si $1-q=0.95, z_{q}=1.65$, y si $1-q=0.99, z_{q}=2.33$. Si se definen $\mu_{d}=\mu / 360$ y $\sigma_{d}=\sigma / \sqrt{360}$ como el rendimiento y la volatilidad diarios, se tiene que

$$
\mathrm{VaR}_{1-q}^{X}=z_{q} \sigma_{d} \sqrt{T-t}-\mu_{d}(T-t),
$$

y, en este caso, $T-t$ toma los valores en días.

\subsection{Valor en riesgo de un bono cupon cero con tasa corta conducida por el modelo de Vasicek}

A continuación se deduce la ecuación matemática que permite calcular el valor en riesgo de un bono cupon cero con la tasa corta guiada por una ecuación diferencial estocástica del modelo de Vasicek. Asimismo, se presenta un aplicación de dicha ecuación a Cetes a 28 días cuyos parámetros, $a, b$ y volatilidad constante $\sigma$, fueron calculados anteriormente para el periodo del 3 de mayo de 2004 al 8 de marzo de 2006. En particular se calcula un VaR a diez días.

\subsubsection{Valor en riesgo y el modelo de Vasicek}

Recordemos que en el modelo de Vasicek la tasa corta, $r_{t}$, está dada por la ecuación (3), es decir tiene la siguiente dinámica estocástica

$$
\mathrm{d} r_{t}=a\left(b-r_{t}\right) \mathrm{d} t+\sigma \mathrm{d} W_{t}
$$

donde $a, b$ y $\sigma$ son constantes positivas y conocidas, y $W_{t}$ es un movimiento Browniano definido en un espacio de probabilidad fijo equipado con su filtración aumentada, $\left(\Omega, \mathcal{F},\left(\mathcal{F}_{t}\right)_{t \in[0, T]}, \mathbb{P}\right)$. Claramente, si $r_{0}$ es constante, $r_{t}$ se distribuye Normal con media

$$
\mathrm{E}\left[r_{t}\right]=r_{0} e^{-a t}+b\left(1-e^{-a t}\right)
$$

y varianza

$$
\operatorname{Var}\left[r_{t}\right]=\sigma^{2} \int_{0}^{t} e^{-2 a(t-s)} d s=\frac{\sigma^{2}}{2 a}\left(1-e^{-2 a t}\right) .
$$


Por otra parte, el precio de un bono cupón cero se obtiene descontando el nominal, en este caso una unidad monetaria, con el promedio de los valores futuros de la tasa corta, es decir,

$$
B\left(r_{t}, t\right)=\mathrm{E}\left\{\exp \left(-\int_{t}^{T} r_{s} \mathrm{~d} s\right) \mid \mathcal{F}_{t}\right\} .
$$

Defina ahora

$$
I(t, T)=\int_{t}^{T} r_{s} \mathrm{~d} s
$$

A continuación se verá que $I(t, T)$ es normal. Por un lado, en virtud del modelo de Vasicek en (58), se sigue que

$$
\int_{t}^{T} \mathrm{~d} r_{s}=a b(T-t)-a \int_{t}^{T} r_{s} \mathrm{~d} s+\sigma \int_{t}^{T} \mathrm{~d} W_{s} .
$$

En consecuencia,

$$
I(t, T)=-\frac{1}{a}\left(r_{T}-r_{t}\right)+b(T-t)+\frac{\sigma}{a} \int_{t}^{T} \mathrm{~d} W_{s} .
$$

Por otro lado, del mismo modelo de Vasicek se tiene que si en (61) se sustituye 0 por $t$ y $t$ por $T$, es decir, se cambia de solución con otro valor final, entonces

$$
r_{T}=r_{t} e^{-a(T-t)}+b\left(1-e^{-a(T-t)}\right)+\sigma \int_{t}^{T} e^{-a(T-s)} \mathrm{d} W_{s} .
$$

Por lo tanto,

$$
r_{T}-r_{t}=\left(b-r_{t}\right)\left(1-e^{-a(T-t)}\right)+\sigma \int_{t}^{T} e^{-a(T-s)} \mathrm{d} W_{s} .
$$

A partir de (62) y (63), se tiene que

$$
I(t, T)=b(T-t)+\left(r_{t}-b\right)\left(\frac{1-e^{-a(T-t)}}{a}\right)+\sigma \int_{t}^{T}\left(\frac{1-e^{-a(T-s)}}{a}\right) \mathrm{d} W_{s} .
$$

Por lo tanto,

$$
\begin{aligned}
\frac{\mathrm{d} B}{\mathrm{~d} r_{t}} & =\mathrm{E}\left\{-\frac{\mathrm{d} I}{\mathrm{~d} r_{t}} e^{-I} \mid \mathcal{F}_{t}\right\} \\
& =-\left(\frac{1-e^{-a(T-t)}}{a}\right) B .
\end{aligned}
$$

Así,

$$
\frac{\mathrm{d} B}{B}=-\left(\frac{1-e^{-a(T-t)}}{a}\right) \mathrm{d} r_{t}
$$


Es decir, $\mathrm{d} B / B$ sigue una distribución normal con

$$
\mathrm{E}\left[\frac{\mathrm{d} B}{B}\right]=-\left(\frac{1-e^{-a(T-t)}}{a}\right) a\left(b-r_{t}\right) \mathrm{d} t .
$$

y

$$
\operatorname{Var}\left[\frac{\mathrm{d} B}{B}\right]=\left(\frac{1-e^{-a(T-t)}}{a}\right)^{2} \sigma^{2} \mathrm{~d} t
$$

De esta manera,

$$
\operatorname{VaR}_{1-q}^{\mathrm{d} B / B}=z_{q} D(t, T) \sigma \sqrt{\mathrm{d} t}+D(t, T) a\left(b-r_{t}\right) \mathrm{d} t
$$

donde

$$
D(t, T)=\frac{1-e^{-a(T-t)}}{a} .
$$

Si desea utilizar la ecuación (10), como alternativa para calcular el VaR de un bono cupón cero, observe que la curva de rendimiento está dada por

$$
\begin{aligned}
R(t, T)= & r_{t} \frac{1-e^{-a(T-t)}}{a(T-t)}-\left(\frac{1-e^{-a(T-t)}}{a(T-t)}-1\right)\left(b-\frac{\sigma^{2}}{2 a^{2}}\right) \\
& +\frac{\sigma^{2}\left(1-e^{-a(T-t)}\right)^{2}}{4 a^{3}(T-t)} .
\end{aligned}
$$

Por lo que,

$$
\mathrm{d} R=\frac{1-e^{-a(T-t)}}{a(T-t)} \mathrm{d} r_{t}
$$

Por lo tanto,

$$
\mu_{\mathrm{d} R}=\mathrm{E}[\mathrm{d} R]==\left(\frac{1-e^{-a(T-t)}}{a(T-t)}\right) a\left(b-r_{t}\right) \mathrm{d} t
$$

y

$$
\sigma_{\mathrm{d} R}^{2}=\operatorname{Var}[\mathrm{d} R]=\left(\frac{1-e^{-a(T-t)}}{a(T-t)}\right)^{2} \sigma^{2} \mathrm{~d} t
$$

De esta manera,

$$
\begin{aligned}
\operatorname{VaR}_{1-q}^{\mathrm{d} B / B} & =z_{q} \gamma \sigma_{\mathrm{d} R}+\gamma \mu_{\mathrm{d} R} \\
& =z_{q} D(t, T) \sigma \sqrt{\mathrm{d} t}+D(t, T) a\left(b-r_{t}\right) \mathrm{d} t,
\end{aligned}
$$

y como era de esperarse se tiene plena coincidencia con (66).

\subsubsection{Valor en riesgo de un bono cupon cero y el modelo de Vasicek}

Utilizando los valores de $a, b$ y volatilidad constante $\sigma$, calculados anteriormente para el periodo del 3 de mayo de 2004 al 8 de marzo de 2006 . Se tiene que, el valor en riesgo de un cete (a 28 días) en los proximos 10 días es de

$$
\operatorname{VaR}_{1-q}^{\mathrm{d} B / B}=0.000000282 .
$$

Como se puede observar es una primera aproximación, por lo que es conveniente utilizar otras metodologías y poder comparar el resultado obtenido. 


\section{Simulación Monte Carlo}

En esta sección se presenta la simulación de la tasa corta con los modelos de Vasicek y Cox Ingersoll y Ross por medio de simulación Monte Carlo. Asimismo, se calcula el precio de un bono cupon cero y el valor en riesgo de Bonos cupon cero.

\subsection{Precio de bono cupón cero}

El precio de bono cupón cero que se coloca en $t$ y que al vencimiento $T$ paga una unidad monetaria está dado por

$$
B(t, T)=e^{-R(t, T)(T--t)},
$$

donde

$$
R(t, T)=\frac{1}{T-t} \int_{t}^{T} f(t, s) d s
$$

Sustituyendo (70) en (71), se tiene

$$
B(t, T)=e^{-\frac{1}{T-t} \int_{t}^{T} f(t, s) d s(T-t)} .
$$

En forma discreta, el precio del bono es

$$
B(t, T)=\exp \left(-\frac{1}{T-t} \sum_{s=t}^{T} r_{s}(T-t)\right) .
$$

donde $r_{s}$ es la tasa corta, es decir la de menor plazo. Para calcular el precio del bono cupon cero, se utilizara la tasa de rendimiento de cetes a 28 días para el periodo 3 de mayo de 2004 al 8 de marzo de 2006.

\subsection{Algoritmo de Simulación}

En esta sección se describe el algoritmo de simulación para determinar el precio de un bono cupon cero donde la dinámica de la tasa corta es guiada por el modelo de Vasicek y Cox-Ingersoll-Ross.

1.) Simular el comportamiento de la tasa corta. Para ello, se generan números aleatorios que siguen una, caminata aleatoria, una determinada distribución, en particular una distribución de tipo normal. La distribución normal se puede generar a partir del método Box-Muller, es decir

$$
X=\sqrt{-2 \ln U_{1}} \cos \left(2 \pi U_{2}\right)
$$

$\mathrm{y}$

$$
Y=\sqrt{-2 \ln U_{1}} \operatorname{sen}\left(2 \pi U_{2}\right) .
$$

Cualquiera de las $X, Y$ genera una distribución normal. Se considera la dinámica de la tasa corta, la cual es conducida por una ecuación diferencial estocástica como en los modelos de Vasicek y CIR. Se utiliza el valor de la tasa corta del día de hoy $r_{0}$ y se continua hasta la fecha de expiración 
del bono cupon cero. Esto da una realización de una trayectoria de la tasa corta.

2.) Para cada realización o trayectoria de la tasa corta se toma la suma de las tasas cortas simuladas y se calcula el promedio de la tasa corta para esta. trayectoria.

3.) Se calcula el precio del bono con esta trayectoria, obteniendo así un valor $B_{1}$.

4.) Repetir $n$ veces las realizaciones sobre el mismo periodo de tiempo y calcular el precio del bono para cada realización o trayectoria, es decir se obtienen $B_{1}, B_{2}, \ldots, B_{n}$.

5.) Se calcula el promedio de los valores $B_{i}$, con $i=1, \ldots, n$, obtenidos por la simulación y de esta manera se obtiene el precio del bono cupon cero. Note que, entre mayor sea el número de simulaciones o de realizaciones, mejor será la precisión del resultado. Si se aumenta en cien veces las simulaciones, entonces aumenta un decima de corrección.

Cabe señalar, que la precisión también depende de la calidad de los números aleatorios propuestos por la diferentes técnicas por lo que es recomendable hacer una prueba de aleatoriedad.

A continuación se aplica el algoritmo para calcular el precio de un bono cupon cero en la que la dinámica de la tasa corta es guiada por una ecuación diferencial estocástica de Vasicek.

\subsection{Simulación del precio de un bono cupón cero con el modelo de Vasicek}

La dinámica de la tasa corta del modelo de Vasicek está dado por la ecuación (3) y se puede escribir en forma discreta como

$$
r_{t+\Delta t}=r_{t}+a\left(b-r_{t}\right) \Delta t+\sigma W_{t},
$$

donde $W_{t}=\sqrt{\Delta t} \varepsilon_{t}$ y $\varepsilon_{t}$ es una normal $\mathcal{N}(0,1)$. A partir del último valor de $r_{t}$ se cálcula $r_{t+\Delta t}$ y se obtiene en forma recursiva el valor de $r_{t+\Delta t}$. El método es fácil de aplicar a una ecuación diferencial estocástica y tiene un error de $O(\delta t)$. El algoritmo mencionado en la sección anterior puede implementarse en cualquier lenguaje de programación cuando el número de simulaciones es del orden de 10,000 (multiplicado por el número de factores o activos a simular), o en una hoja de excel cuando el número de simulaciones es más o menos rápido, es decir calculado en unos cuantos nanosegundos o segundos. Se necesita solamente proponer los valores de: la tasa corta de interés al día de hoy, $r_{0}$, la volatilidad, incrementos en el tiempo y para $\varepsilon_{t}$ una muestra de variables aleatorias que se distribuyan como normal estandarizada.

A continuación se presenta la gráfica de simulaciones Monte Carlo para el modelo de Vasicek: 


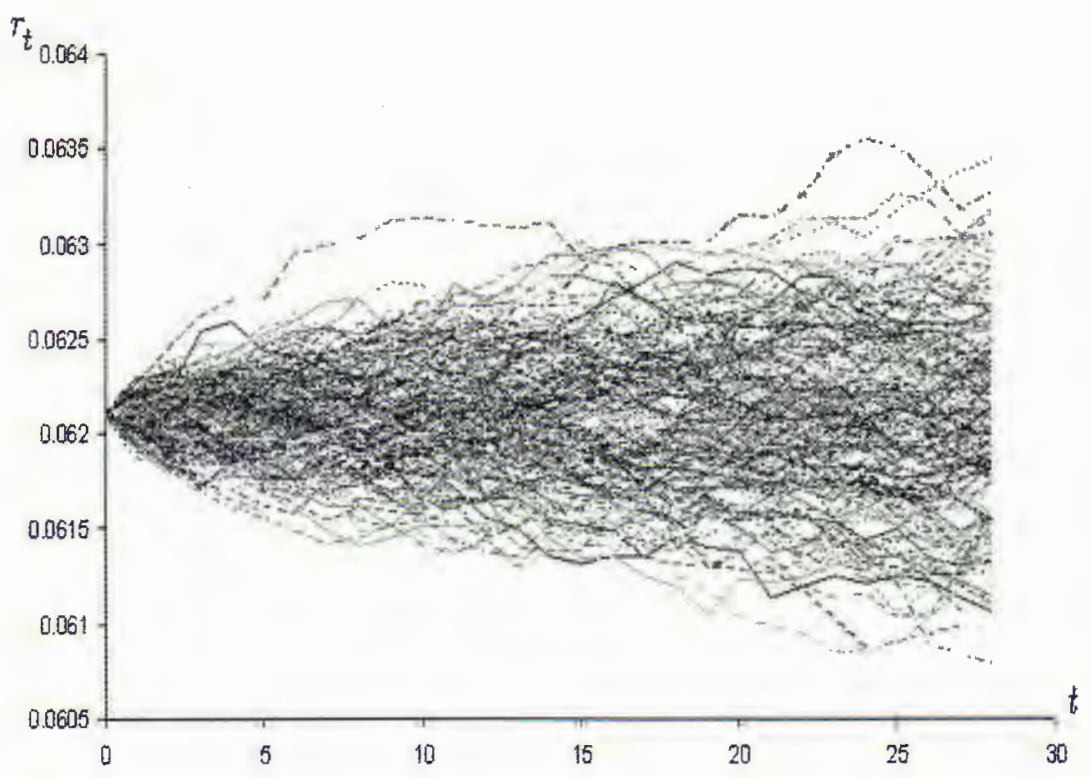

Gráfica 11. Simulación Monte Carlo de la tasa corta, del modelo de Vasicek, para valuar bonos cupon cero, cetes a 28 días.

En la siguiente tabla se presenta el precio del bono cupon cero calculado por el método de simulación Monte Carlo, cuyas trayectorias simuladas de la tasa corta aparecen en la gráfica anterior, 4.2. En este cálculo se utilizó la tasa de rendimiento de Bonos cupon cero (Cetes) a 28 días en el periodo del 3 de mayo de 2004 al 8 de marzo de 2006.

Tabla 3. Precio del Bono cupón cero determinado por simulación Monte Carlo.

\begin{tabular}{|c|c|c|}
\hline \multicolumn{3}{|c|}{$\begin{array}{c}\text { Simulación de la tasa corta del modelo de } \\
\text { Vasicek por el método de Monte Carlo }\end{array}$} \\
\hline \multicolumn{2}{|c|}{ Precio del Bono cupon cero $B\left(r_{t}, t, T\right)=0.995142$} \\
\hline Plazo del Bono & $T=28$ dias & $\mathrm{a}=0.007032413$ \\
\hline Volatilidad & $0.090579173 \%$ & $\mathrm{~b}=0.083772223$ \\
\hline $\begin{array}{c}\text { Tasa de interés } \\
\text { libre de riesgo } \\
\text { sin impuesto }\end{array}$ & $r_{0}=6.021 \%$ & VaR $=0.029$ \\
\hline
\end{tabular}

Fuente: Valmer. 
El precio del Bono cupón cero corresponde a 10,000 simulaciones o trayectorias de la tasa corta y que aparecen sólo 200 de éstas en la gráfica 11.

\subsection{Dinámica de la tasa corta y saltos Poisoon}

Ahora, se supone que el número de saltos, movimientos extremos y repentinos, en la tasa corta, por unidad de tiempo, siguen un proceso de Poisson $N_{t}$ con intensidad $\lambda$, de tal manera que

$$
\mathbb{P}^{(N)}\{\text { un salto unitario durante } \mathrm{d} t\}=\mathbb{P}^{(N)}\left\{\mathrm{d} N_{t}=1\right\}=\lambda \mathrm{d} t+o(\mathrm{~d} t),
$$

mientras que

$$
\mathbb{P}^{(N)}\{\text { ningún salto en } \mathrm{d} t\}=\mathbb{P}^{(N)}\left\{\mathrm{d} N_{t}=0\right\}=1-\lambda \mathrm{d} t+o(\mathrm{~d} t),
$$

donde $o(\mathrm{~d} t) / \mathrm{d} t \rightarrow 0$ cuando $\mathrm{d} t \rightarrow 0$. Así,

$$
\mathrm{E}^{(N)}\left[\mathrm{d} N_{t}\right]=\operatorname{Var}^{(N)}\left[\mathrm{d} N_{t}\right]=\lambda \mathrm{d} t .
$$

El número inicial de saltos se supone igual a cero, es decir, $N_{0}=0$.

Considere ahora un movimiento Browniano $\left(Z_{t}\right)_{t \geq 0}$ definido en un espacio de probabilidad fijo con su filtración aumentada $\left(\Omega^{(\bar{Z})}, \mathcal{F}^{(Z)},\left(\mathcal{F}_{t}^{(Z)}\right)_{t \geq 0}, \mathbb{P}^{(Z)}\right)$. Se supone que la tasa corta sigue un proceso de la forma:

$$
\mathrm{d} r_{t}=a\left(b-r_{t}\right) \mathrm{d} t+\sigma \mathrm{d} W_{t}+\eta \mathrm{d} N_{t},
$$

donde $a, b, \mathrm{y} \sigma$ son constantes positivas, $\sigma$ es la volatilidad instantánea de la tasa corta, $\eta$ es el tamaño medio esperado de un salto, de Poisson, en la tasa corta y $\left\{W_{t}\right\}_{t \geq 0}$ es un movimiento Browniano definido sobre un espacio fijo de probabilidad $(\Omega, \mathcal{F}, \mathbb{P})$ junto con su filtración aumentada $\mathbb{F}=\left\{\mathcal{F}_{t}\right\}_{t \geq 0}$, entonces se establece una de las clases más importantes de modelos de tasa corta con reversión a la media y parámetros constantes. El proceso $W_{t}$ se supone independiente de $N_{t}$. En lo que sigue, $\sigma, \lambda$ y $\eta$ son constantes positivas. Se supone que $\mathrm{d} W_{t}$ es una variable aleatoria distribuida normalmente con $\mathrm{E}\left[\mathrm{d} W_{t}\right]=0$ y $\operatorname{Var}\left[\mathrm{d} W_{t}\right]=\mathrm{d} t$. Los procesos estocásticos $\mathrm{d} W_{t} \mathrm{y} \mathrm{d} N_{t}$ se suponen no correlacionados, es decir,

$$
\operatorname{Cov}\left(\mathrm{d} W_{t}, \mathrm{~d} q_{t}\right)=0
$$

A continuación se presenta la gráfica de simulaciones Monte Carlo para el modelo de Vasicek: 


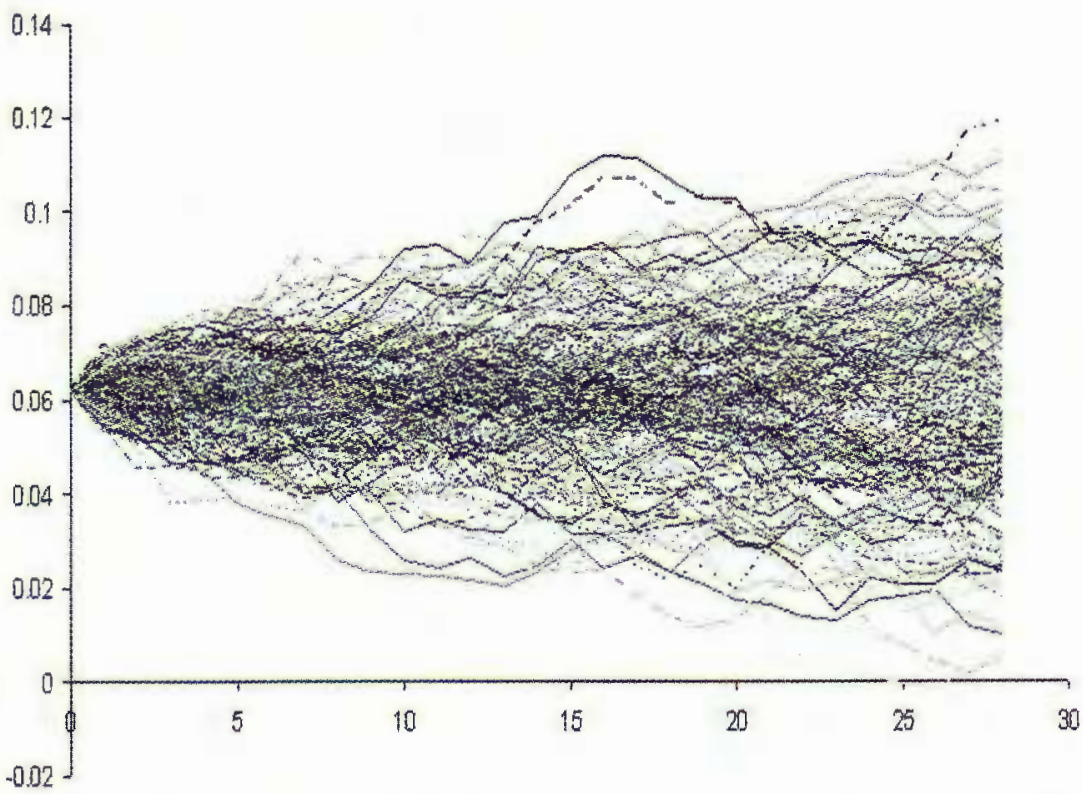

Gráfica 12. Simulación de la tasa corta del modelo de Vasicek por el método de Monte Carlo, para valuar bonos cupon cero.

En la siguiente tabla se presenta el precio del bono cupon cero calculado por el método de simulación Monte Carlo, cuyas trayectorias simuladas de la tasa corta aparecen en las gráfica anterior, 4.3. En este cálculo se utilizó la tasa de rendimiento de Bonos cupon cero (Cetes) a 28 días, diaria, en el periodo del 3 de mayo de 2004 al 8 de marzo de 2006.

Tabla 4. Precio del Bono cupón cero determinado por simulación Monte Carlo.

\begin{tabular}{|c|c|c|}
\hline \multicolumn{3}{|c|}{ Simulación de la tasa corta del modelo } \\
de Vasicek con saltos de Poisson, por el métodode Monte Carlo \\
\hline \multicolumn{2}{|c|}{ Precio del Bono cupon cero $B\left(r_{t}, t, T\right)=0.995288$} \\
\hline Plazo del Bono & $T=28$ dias & $\mathrm{a}=0.007032413$ \\
\hline Volatilidad & $0.090579173 \%$ & $\mathrm{~b}=0.083772223$ \\
\hline $\begin{array}{c}\text { Tasa de interés } \\
\text { libre de riesgo } \\
\text { sin impuesto }\end{array}$ & $r_{0}=6.021 \%$ & $\lambda=0.002092$ \\
& $\eta=0.002236$ \\
\hline
\end{tabular}

Fuente: Valmer.

Los valores de $\lambda$ y $\eta$ fueron determinados de los rendimientos de las tasas de CETES a 28 días cuyo valor de un dia a otro supero un incremento en la tasa por arriba del $2 \%$ y por abajo del $5 \%$, que se considero importante. En este caso 
se presentaron 3 eventos (tasas) que cayeron en este intervalo de un conjunto de 478 observaciones para el periodo del 3 de mayo de 2004 al 8 de marzo de 2006 y del cual se tomo un promedio en dicha tasa y tomando como punto inicial $r_{0}$ del último dato observado.

\subsection{Simulación del precio de un bono cupón cero con el modelo de CIR}

La dinámica de la tasa corta del modelo de CIR está dado por la ecuación (18) $y$ se puede escribir en forma discreta $a^{5}$ como

$$
r_{t+\Delta t}=r_{t}+a\left(b-r_{t}\right) \Delta t+\sigma \sqrt{r_{t}} W_{t}
$$

donde $W_{t}=\sqrt{\Delta t} \varepsilon_{t}$ y $\varepsilon_{t}$ es una normal $\mathcal{N}(0,1)$ y $r_{t} \sim \chi^{2}(n, \delta)$, donde $n$ representa los grados de libertad y $\delta$ el parámetro de no centralidad. A partir del último valor de $r_{t}$ se cálcula $r_{t+\Delta t}$ y se obtiene en forma recursiva el valor de $r_{t+\Delta t}$. El método es fácil de aplicar a una ecuación diferencial estocástica y tiene un error de $O(\delta t)$. Se necesita solamente conocer los valores de: la tasa corta de interés al día de hoy, $r_{0}$, lá volatilidad, incrementos en el tiempo y para $\varepsilon_{t}$ una muestra de variables aleatorias que se distribuyan como normal estandarizada, $r_{t}$ puede ser calculado a partir de la distribución $\chi^{2}(n, \delta)$ a un nivel de significancia $\alpha$.

A continuación se presenta la gráfica de simulaciones Monte Carlo para el modelo de CIR:

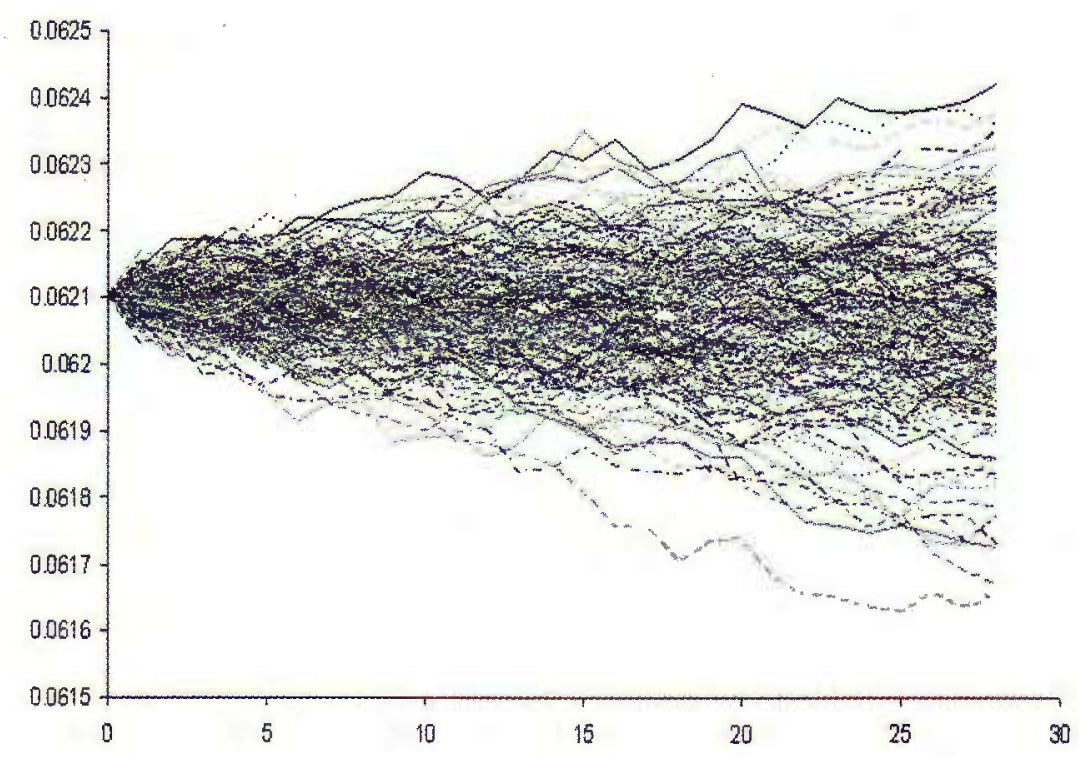

Gráfica 13. Simulación Monte Carlo con el modelo CIR.

5 Vea: Jessica James and Webber (2000). 
En la siguiente tabla se presenta el precio del bono cupon cero calculado por el método de simulación Monte Carlo, cuyas trayectorias simuladas de la tasa corta aparecen en las gráfica anterior, 13. En este cálculo se utilizo la tasa de rendimiento de Bonos cupon cero (Cetes) a 28 días en el periodo de 3 de mayo de 2004 al 8 de marzo de 2006

Tabla 5. Precio del Bono cupón cero determinado por simulación Monte Carlo del modelo CIR.

\begin{tabular}{|c|c|c|}
\hline \multicolumn{3}{|c|}{$\begin{array}{c}\text { Simulación de la tasa corta del modelo Monte Carlo } \\
\text { del modelo CIR, por el método de Monte Carlo }\end{array}$} \\
\hline \multicolumn{2}{|c|}{ Precio del Bono cupon cero $B\left(r_{t}, t, T\right)=0.995183$} \\
\hline Plazo del Bono & $T=28$ dias & $\mathrm{a}=0.005900$ \\
\hline Volatilidad & $0.076242 \%$ & $\mathrm{~b}=0.08800$ \\
\hline $\begin{array}{c}\text { Tasa de interés } \\
\text { libre de riesgo } \\
\text { sin impuesto }\end{array}$ & $r_{0}=6.021 \%$ & $\mathrm{VaR}=0.012, \mathrm{VaR}=0.017$ \\
\hline
\end{tabular}

Fuente: Valmer.

El precio del Bono cupón cero corresponde a 10,000 simulaciones o trayectorias de la tasa corta y que aparece sólo 200 de éstas en la gráfica 13.

\subsection{Dinámica de la tasa corta y saltos Poisoon, CIR}

Ahora, se supone que el número de saltos, movimientos extremos y repentinos, en la tasa corta, por unidad de tiempo, siguen un proceso de Poisson $N_{t}$ con intensidad $\lambda$, de tal manera que

$$
\mathbb{P}^{(N)}\{\text { un salto unitario durante } \mathrm{d} t\}=\mathbb{P}^{(N)}\left\{\mathrm{d} N_{t}=1\right\}=\lambda \mathrm{d} t+o(\mathrm{~d} t),
$$

mientras que

$$
\mathbb{P}^{(N)}\{\text { ningún salto en } \mathrm{d} t\}=\mathbb{P}^{(N)}\left\{\mathrm{d} N_{t}=0\right\}=1-\lambda \mathrm{d} t+o(\mathrm{~d} t),
$$

donde $o(\mathrm{~d} t) / \mathrm{d} t \rightarrow 0$ cuando $\mathrm{d} t \rightarrow 0$. Así,

$$
\mathrm{E}^{(N)}\left[\mathrm{d} N_{t}\right]=\operatorname{Var}^{(N)}\left[\mathrm{d} N_{t}\right]=\lambda \mathrm{d} t .
$$

El número inicial de saltos se supone igual a cero, es decir, $N_{0}=0$.

Considere ahora un movimiento Browniano $\left(Z_{t}\right)_{t \geq 0}$ definido en un espacio de probabilidad fijo con su filtración aumentada $\left(\Omega^{(\bar{Z})}, \mathcal{F}^{(Z)},\left(\mathcal{F}_{t}^{(Z)}\right)_{t \geq 0}, \mathbb{P}^{(Z)}\right)$. Se supone que la tasa corta sigue un proceso de la forma:

$$
\mathrm{d} r_{t}=a\left(b-r_{t}\right) \mathrm{d} t+\sigma \sqrt{r_{t}} \mathrm{~d} W_{t}+\eta \mathrm{d} N_{t}
$$

donde $a, b$, y $\sigma$ son constantes positivas, $\sigma$ es la volatilidad instantánea de la tasa corta, $\eta$ es el tamaño medio esperado de un salto, de Poisson, en la tasa 
corta y $\left\{W_{t}\right\}_{t \geq 0}$ es un movimiento Browniano definido sobre un espacio fijo de probabilidad $(\Omega, \mathcal{F}, \mathbb{P})$ junto con su filtración aumentada $\mathbb{F}=\left\{\mathcal{F}_{t}\right\}_{t>0}$, entonces se establece una de las clases más importantes de modelos de tasa corta con reversión a la media y parámetros constantes. El proceso $W_{t}$ se supone independente de $N_{t}$. Como antes, $\sigma, \lambda$ y $\eta$ son constantes positivas. Se supone que $\mathrm{d} W_{t}$ es una variable aleatoria distribuida normalmente con $\mathrm{E}\left[\mathrm{d} W_{t}\right]=0 \mathrm{y}$ $\operatorname{Var}\left[\mathrm{d} W_{t}\right]=\mathrm{d} t$. Los procesos estocásticos $\mathrm{d} W_{t} \mathrm{y} \mathrm{d} N_{t}$ se suponen no correlacionados, es decir,

$$
\operatorname{Cov}\left(\mathrm{d} W_{t}, \mathrm{~d} q_{t}\right)=0 \text {. }
$$

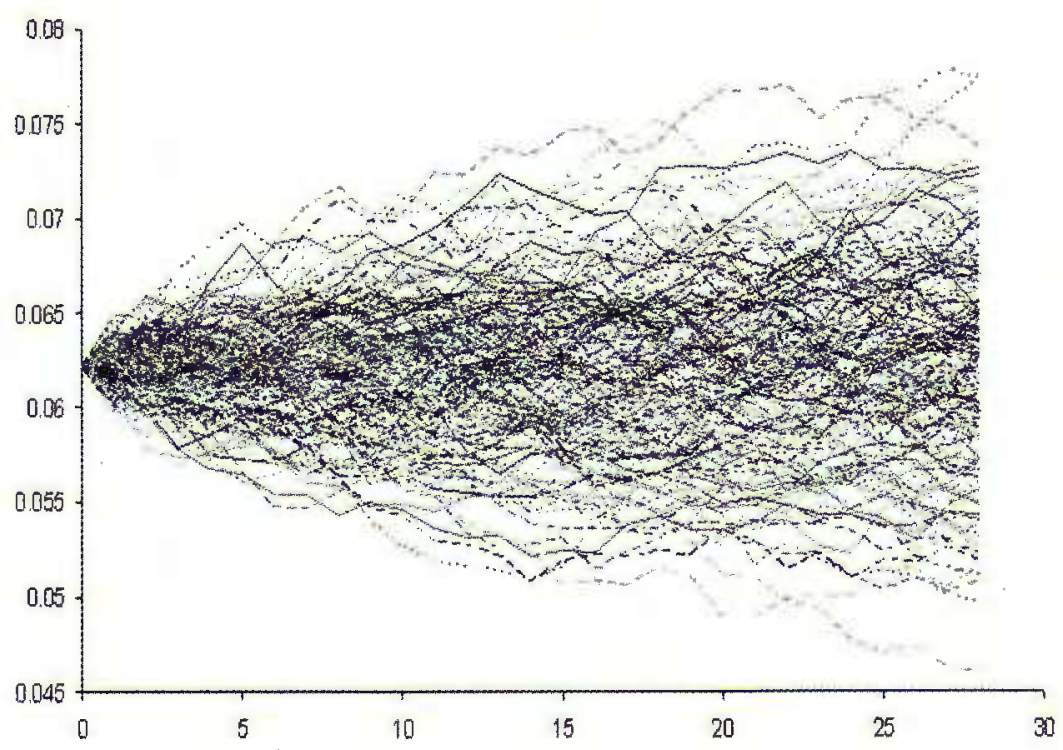

Gráfica 14. Simulación Monte Carlo con el modelo CIR.

Tabla 6. Precio del Bono cupón cero determinado por simulación Monte Carlo del modelo CIR.

\begin{tabular}{|c|c|c|}
\hline \multicolumn{3}{|c|}{$\begin{array}{c}\text { Simulación Monte Carlo } \\
\text { del modelo CIR con Poisson }\end{array}$} \\
\hline Precio del Bono cupon cero $B\left(r_{t}, t, T\right)=0.995186$ \\
\hline Plazo del Bono & $T=28$ dias & $\mathrm{a}=0.005900$ \\
\hline Volatilidad & $0.0076242 \%$ & $\mathrm{~b}=0.008800$ \\
\hline Tasa de interés & & $\lambda=0.002092$ \\
libre de riesgo & $r_{0}=6.021 \%$ & $\eta=0.002236$ \\
sin impuesto & & \\
\hline
\end{tabular}

Fuente: Valmer. 
Cox, J., J. Ingersoll, and S. Ross (1985a). An Intertemporal General Equilibrium Model of Asset Prices. Econometrica, 53(2), pp. 363-384.

Cox, J., J. Ingersoll, and S. Ross (1985b). A Theory of the Term Structure of Interest Rates. Econometrica, 53(2), pp. 385-408.

Churchill, V. and J. Brown (1978). Fourier Series and Boundary Value Problems. Third ed., Mc Graw Hill.

Dothan, U. (1978). On the term estructure of interest rates. Journal of Financial Economics 6, pp. 59-69.

Dufie, D. and J. Pan. (1997). An overview of value at risk.Journal of Derivatives, 4(3), pp. 7-49.

Evans, G., J. Blackledge, and Yardley P (1986). Analytical Methods for Partial Differential Equations. Springer.

Embrechts, P., C. Klüppelberg, and T. Mikosch (1999). Modelling extremal events for insurance and finance, Springer.

Goldberg, D. (1999). Genetic Algorithms in Search, Optimization and Machine Learning, Addison Wesley.

Greene, W. (1997). Econometric Analysis, 3a edición, Prentice Hall.

Handscomb, D. (1965). Methods of Numerical Approximation, Pergamon Press.

Ho, T. and S. Lee (1986). Term Structure Movements and Pricing of Interest Rtae Claims, Journal of Finance, 41, pp. 1011-1029.

Hull, J. (2003). Options, Futures and other Derivatives, 5th edition, Prentice Hall.

Hull, J. and A. White (1996). Pricing Interest-Rate-Derivative Securities, Review of Financial Studies, 3, pp. 573-592.

Ferson, E. (1986). The Empirical Implications of the Cox, Ingersoll, Ross Theory of the Term Structure of Interest Rates: Discussion. Journal of Finance, 41(3), pp. 630-632.

Jaeckel, P. (2000). Monte Carlo methods in Finance, Wiley Finance.

Janke, W. (2002). Pseudo Random Numbers: Generation and Quality Checks, Quantum Simulations of Complex Many-Body Systems: From Theory to Algorithms, Lecture Notes, 10 , pp. 447-458.

James, J. and N. Webber (2000). Interest Rate Modelling. John Wiley \& Sons, LTD.

J. P. Morgan Bank (1995). RiskMetrics Technical Manual. New York.

Johnson, N. (2003). Financial Market Complexity, Oxford University Press.

Johnston, J. and J. DiNardo (1997). Econometric Methods, Mc Graw Hill.

Jorion, P. (2001). Value at Risk: The New Benchmark for Managing Financial Risk. 2nd ed., McGraw-Hill.

Kijima, M. (2003). Stochastic Processes with applications to Finance. CHAPMAN \& HALL/CRC, A CRC Press Company.

Kijima, M. (2003). Stochastic Processes with Aplications to Finance, Chapman \& Hall/CRC, ACRC Press Company.

Knuth, D (1999). The Art of Computer Programming, 2.

Levy, H., M. Levy, and S. Solomon (2000). Microscopic simulation of financial markets: from investor behaviour to market phenomena, Academic Press.

Longstaff, F. and E. Schwartz (1979). Interest Rate volatility and the term structure: A two-factor general equilibrium model, Journal of Finance, 47, pp. 1259-1282.

Macaulay, F. (1938). Some theoretical Problems Suggested by the Movements of Interest Rates, Bond Yield, and Stock Prices in the United States since 18bo. Columbia University Press. New York.

Marsaglia, G. (1984). The Exact-Approximation for Generating Random Variables in a Computer. $79(385)$, pp. 218-221.

Merton, R. (1992). Continuous-Time Finance, Blackwell.

Michalewicz, Z. (1999). Genetic Algorithrns + Data Structures = Evolution Programs, 3a edición, Springer.

Musiela, M. and M. Rutkowsky (1998). Martingale Methods in Financial Modelling, Springer. 
Ross, S. (1999). Simulación. Pearson, Prentice Hall, segunda edición.

Nowman, K. (1997). Gaussian Estimation of Single-Factor Continuous Time Models of the Term Estructure of Interest Rates. The Journal of Finance, LII(4).

Vasicek, O. (1977). An Equilibrium Characterization of the Term Structure. Journal of Financial Economics, 5, pp. 177-188.

Von Neuman, J. and S. Ulam (1949). The Monte Carlo method. J. American Statistical Association, 44(247), pp. 335-341.

Wilmott, P. (1998). Derivatives. The Theory and Practice of Financial Engineering. John Wiley \& Sons, Inc., New York.

Willmott, P. (2000). Paul Willmott on Quantitative Finance, John Wiley \& Sons LTD. 